Document downloaded from:

http://hdl.handle.net/10251/82886

This paper must be cited as:

Gonzalez-Martinez, SR.; Castellanos Hernández, WE.; Guzman Castillo, PF.; Arce Vila, P.; Guerri Cebollada, JC. (2016). Simulation and experimental testbed for adaptive video streaming in ad hoc networks. Ad Hoc Networks. 52:89-105. doi:10.1016/j.adhoc.2016.07.007.

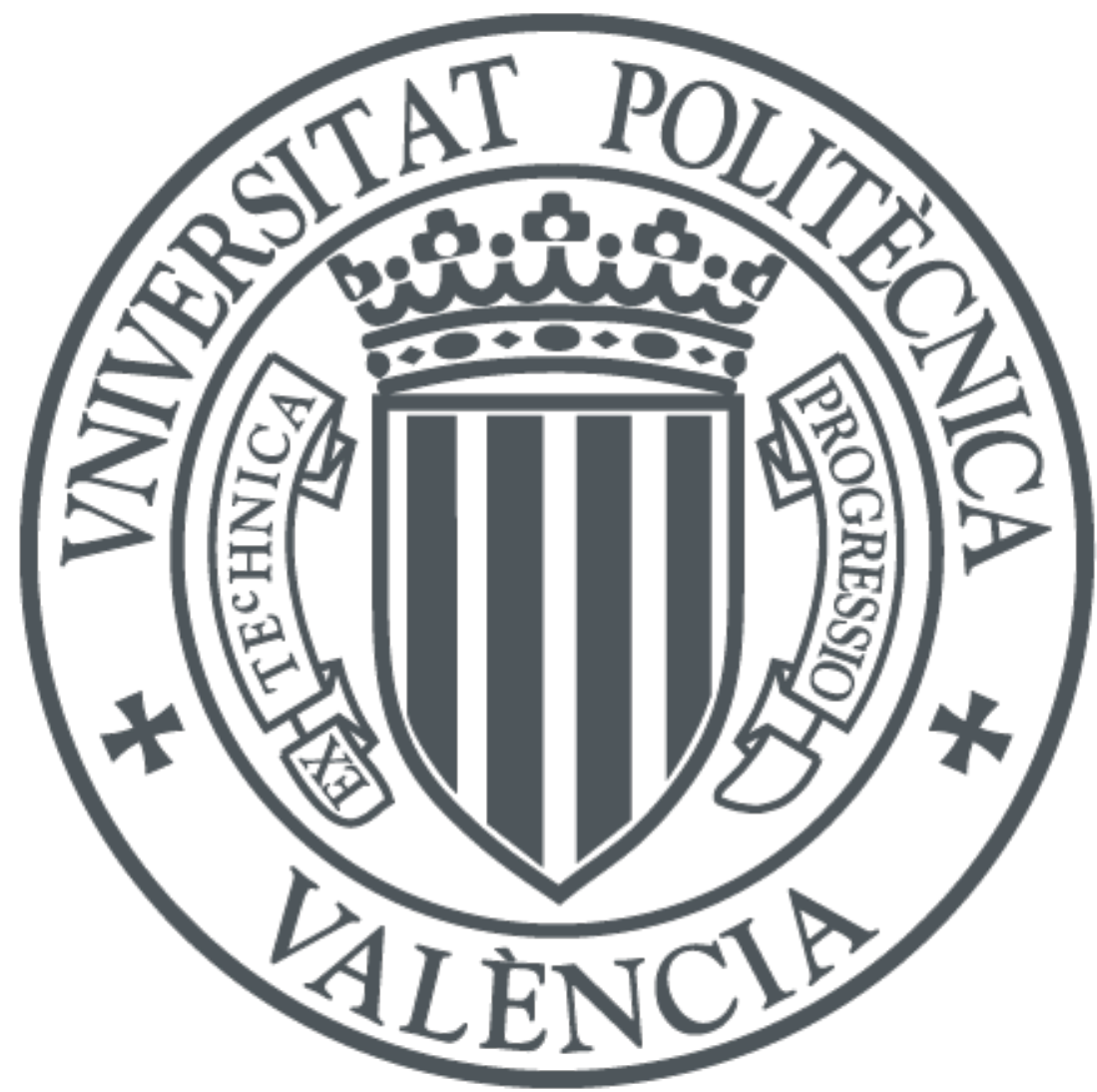

The final publication is available at

http://dx.doi.org/10.1016/j.adhoc.2016.07.007

Copyright Elsevier

Additional Information 


\title{
Simulation and Experimental Testbed for Adaptive Video Streaming in Ad hoc Networks
}

\author{
Santiago González, Wilder Castellanos, Paola Guzmán, Pau Arce, Juan Carlos Guerri*. \\ Institute of Telecommunications and Multimedia Applications (iTEAM) \\ Universitat Politècnica de València, Spain \\ \{sangonma,wilcashe\}@posgrado.upv.es, \{paoguzc1,paarvi\}@iteam.upv.es, jcguerri@dcom.upv.es
}

\begin{abstract}
This paper presents a performance evaluation of the scalable video streaming over mobile ad hoc networks. In particular, we focus on the rate-adaptive method for streaming scalable video (H.264/SVC). For effective adaptation a new cross-layer routing protocol is introduced. This protocol provides an efficient algorithm for available bandwidth estimation. With this information, the video source adjusts its bit rate during the video transmission according to the network state. We also propose a free simulation framework that supports evaluation studies for scalable video streaming. The simulation experiments performed in this study involve the transmission of SVC streams with Medium Grain Scalability (MGS) as well as temporal scalability over different network scenarios. The results reveal that the rate-adaptive strategy helps avoid or reduce the congestion in MANETs obtaining a better quality in the received videos. Additionally, an actual ad hoc network was implemented using embedded devices (Raspberry Pi) in order to assess the performance of the proposed adaptive transmission mechanism in a real environment. Additional experiments were carried out prior to the implementation with the aim of characterizing the wireless medium and packet loss profile. Finally, the proposed approach shows an important reduction in energy consumption, as the study revealed.
\end{abstract}

\section{Categories and Subject Descriptors}

C.2.1 [Computer Systems Organization]: Computer Communications Networks- Network Architecture and Design, wireless communication

\section{General Terms}

Algorithms, Performance, Design; Experiment; Traces. Multi-hop.

\section{Keywords}

Mobile ad hoc networks; SVC video; scalable video streaming; rate-adaptive video streaming; testbed evaluation, experimental platforms.

\section{INTRODUCTION}

The continuous increase in processing capabilities of wireless devices, as well as the improvements in energy efficiency, have motivated a significant increase in the transmission of multimedia content for video on demand services, such as YouTube or Vimeo. Because of this, the development of new network technologies to improve video streaming over wireless networks has had a growing interest. Among the different types of wireless networks, it is expected that Mobile Ad hoc Networks (MANET) will be widely used in the near future, in various settings and under different paradigms [1]. MANETs consists of mobile nodes that are connected via wireless links without using an existing network infrastructure. Therefore, each node has to rely on others to communicate with other nodes outside its transmission range, creating the so-called multi-hop communication. However, the transmission of video over these networks is not an easy task because of the mobility of the nodes and the fact that nodes cannot simultaneously access the shared medium. Moreover, if any rate-adaptive technique is considered during video transmission, received video may suffer significant quality degradation. Hence, new schemes for more flexible and robust transmission should be apply. One possibility is the use of scalable video coding (H.264/SVC, Scalable Video Coding) [2]. A video encoded using H.264/SVC standard is composed of several layers (a base layer and one or more enhancement layers) that provide different levels of quality. With this layered scheme we obtain: i) coded streams with greater robustness in environments with high packet loss and ii) the possibility of adapting the video stream to the network conditions by removing or adding enhancement layers.

In this article, an evaluation of two schemes of scalable video streaming over MANETs was performed. In the first scheme, video is transmitted by means of maintaining a constant transmission rate and sending the information of all layers. The other scheme incorporates an adaptive model in which the source of traffic eliminates layers from SVC stream in order to adapt to the available bandwidth. Two complementary tools for the study of SVC traffic over data networks are also proposed. On the one hand, a new routing protocol with inter-

*Corresponding author.Tel.: +34 963879580; fax: +34 963879583. 
layer communication aims to estimate and inform the source resources available on the network (in terms of available bandwidth). Moreover, a new simulation framework called SVCEval-RA (SVC Evaluation platform for Rate-Adaptive Video) is exposed. This software tool simulates the transmission of scalable video over wireless networks. The results of the simulations prove that the adaptive strategy prevents or helps to reduce network congestion and packet loss. Therefore, transmitted videos have been received with higher quality. Moreover, the design and implementation of a real testbed is presented. This testbed has been used for the experimental evaluation of the proposal in a real environment. The testbed has been built using embedded devices with wireless communications, specifically Raspberry Pi B+ [3]. The experiments have been conducted to assess the adaptive bitrate proposal in a multi-hop scenario. Finally, wireless cards have been characterized regarding energy consumption taking into account the throughput achieved during the regular operation of data transmission of the implemented node.

The paper is organized as follows: Section 2 describes some related work. Section 3 presents a summary of the most important aspects of adaptive scalable video streaming. Section 4 describes the main features of the developed simulation platform. Section 5 gives a brief introduction of the QoS routing protocol used in the studies. In Sections 6 and 7, simulation environment and results are described respectively. Section 8 describes the testbed implementation as well as the experiments carried out and the outcomes obtained. Finally, in Section 9 some conclusions and future lines of work are proposed.

\section{RELATED WORK}

Different SVC-based solutions have been recently proposed for the video streaming. Famaey et al. [4] and Kalva et al. [5] discuss the advantages of using SVC in adaptive video streaming over HTTP versus single layer coding (AVC, Advanced Video Coding). Xiang et al. [6] developed an algorithm for SVC flow adjustment for video streaming over wireless networks using DASH (Dynamic Adaptive Streaming over HTTP) [7].

However, HTTP is not the only alternative for video delivery using SVC. The flexibility and robustness of the layered scheme of scalable video coding has motivated the development of several proposals to integrate SVC with other network technologies. For example, Sanna and Izquierdo [8] reported SVC video streaming over peerto-peer networks with network coding techniques, Alabdulkarim et al. [9] implement a solution for SVC video streaming over ZigBee networks, Yaacoub et al. [10] propose a scheme for improving the scalable video streaming in vehicular networks and in [11] SVC over LTE mobile networks is reported.

Regarding ad hoc mobile networks, Halloush et al. [12] and Rhaiem et al. [13] evaluated the performance of conventional routing protocols for MANETs during transmission of SVC streams. The results of these studies show the need to implement new network mechanisms for achieving Quality of Service (QoS). A solution focused on providing QoS in MANETs for SVC video streaming is presented by Lal et al.[14]. This proposal is based on the implementation of a new routing protocol that includes admission control based on the available bandwidth. There is also the work presented by Radu et al.[15], which introduces the subjective quality monitoring and also a method of error protection for SVC video streaming. To overcome the high packet loss characteristic of MANETs, Lai and Liou [16] propose a system based on the prioritization scheme of video packets. However, the solutions proposed until now for MANETs have not assessed in detail the option of adapting the bit rate, by removing or adding SVC layers, according to the estimation of the available bandwidth. Furthermore, the existing tools for evaluating the developed solutions for SVC streaming (such as EvalSVC [17] and myEvalSVC [18]) do not enable adaptive streaming operation and therefore, the entire SVC stream is always transmitted. This operation mode of the aforementioned evaluation frameworks limits the utilization of the layered schemed of SVC. Thus, the development of a new simulation platform as the one proposed in this article is needed. On the other hand, solutions studied for MANETs only evaluate the temporal and spatial scalability that provides SVC, leaving aside the quality scalability. In this article, not only is the combination of temporal and quality scalability used, but also the MGS (Medium Grain Scalability) scheme, which is the scheme regarding quality scalability that best performance features offers, as evidenced by numerous studies, e.g. [19] and [20]. 
Moreover, although simulations provide considerable advantages, for example, in terms of scalability and costs reduction, the validity of the results obtained in the simulations and the complexity of a real-world implementation of the proposals, remains a subject of discussion in ad hoc networks.

Regarding that, in [21] a detailed analysis of the advantages and disadvantages provided by each methodology is presented. On that score, we believe that an appropriate validation requires experimentation with simulations and real-world equipment. However, the use of real-world equipment involves additional efforts in terms of design, configuration, installation, control and execution of the experiments as well as implementation costs.

Accordingly, the number of available studies applying this methodology is limited, some notable examples include: APE (Ad hoc Protocol Evaluation) [22], it is a study that describes a testbed for evaluating OLSR, AODV, LUNAR under conditions of mobility, nodes are implemented by computers and used a total of 9 devices. J. Yi et al [23], describes a set of experiments to evaluate the operation of a multipath variant of the OLSR protocol, using laptops as ad hoc nodes in a scenario made up of 11 nodes and a maximum of 4 hops. In [24] a testbed developed to assess the range of communication between user devices in Vehicular Ad-Hoc Networks (VANETs) is described. Results describe a maximum connectivity distance of $75 \mathrm{~m}$ using smartphones and 802.11g standard. A characterization of communication links for devices 802.15.4 and 802.11 is presented in [25], using mote platforms and laptops respectively.

In regard to experimentation with a greater level of scalability, [26] describes the project Emulab consisting of a remote laboratory containing hundreds of nodes. The nodes were initially implemented on platforms mote and today experimentation includes $802.11 \mathrm{a} / \mathrm{b} / \mathrm{g}$ devices. However, the access to the laboratory is limited. Moreover, in [27] the authors described QuRiNET, a communication project which simultaneously serves as a Wireless Mesh Networks testbed. The project consists of a set of 30 nodes carried out using embedded platforms Soekris net4826 and deployed in an area of 800 hectares. Similarly, DOME (Diversity Outdoor Mobile Environmet) [28] is a testbed that implements mesh nodes on buses for the study of mobility in such networks. Moreover, a more limited set of studies that implement testbed are focused on the evaluation of energy consumption. Specifically, [29] presents a characterization of energy consumption in mobile phones during the operation of the interface 802.11. In [30] a study of energy consumption made directly on the wireless card using measuring instruments is described. Finally, [31] presents a framework for monitoring energy expenditure using development platforms.

\section{ADAPTIVE SCALABLE VIDEO STREAMING}

There are two main methods for video delivery: traditional video streaming (that includes simple progressive download and real time streaming techniques) and adaptive streaming. In the traditional techniques, video is sent as a flow of packets using a protocol that is in charge of establishing a session between the video provider and the client. This technique is known as a real time streaming. Also, the progressive download technique is considered as traditional video transmission technique. In this technique users request video content which is downloaded progressively into a local buffer. As soon as there are sufficient data the media starts to play. If the playback rate exceeds the download rate, then playback is delayed until more data is downloaded. However, these traditional video transmission schemes lack of the necessary flexibility and adaptability since the video file is delivered to the end-user with a constant bit rate through a persistent source-destination connection. Thus, if a particular transmission channel experiences congestion, the quality of the video can significantly degrade (see Figure 1).

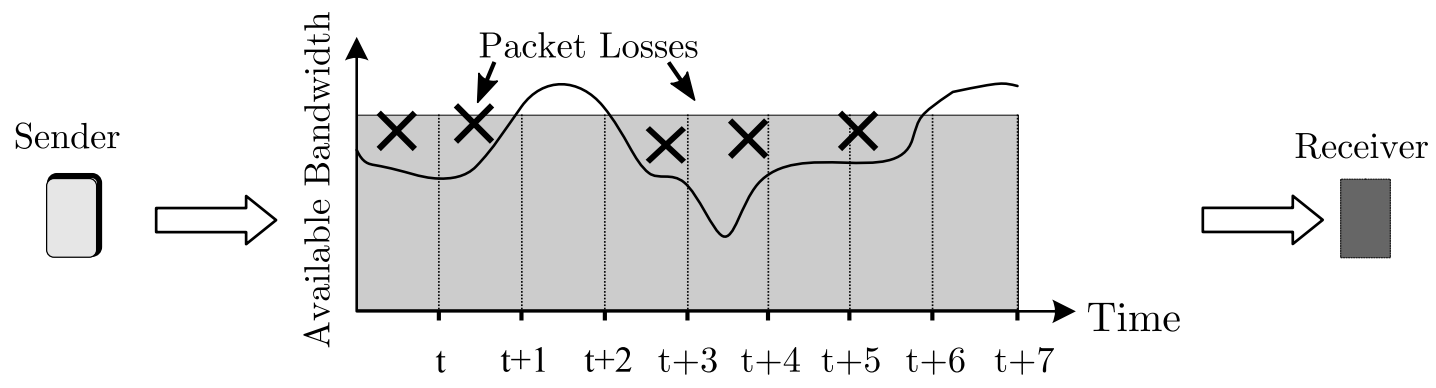

Figure 1. Traditional video streaming. 
Adaptive streaming [32],[33],[34] is a technique which estimates the available bandwidth and CPU capacity in order to adapt the video quality that is provided to the user. To implement the idea of adaptive transmission, three types of techniques have been proposed. One possibility is transcoding, which consists on transcode from one encoding to another to match a specific bit rate. However, there are some important disadvantages as the high cost of transcoding due to the fact that the raw video content requires to be adapted several times for different quality requests. Consequently, scalability decreases since transcoding needs to be performed for every different client. Therefore, because of lack of flexibility and the high computational requirements of a real-time transcoding system, the transcoding technique is not a suitable solution for mobile ad hoc networks [35].

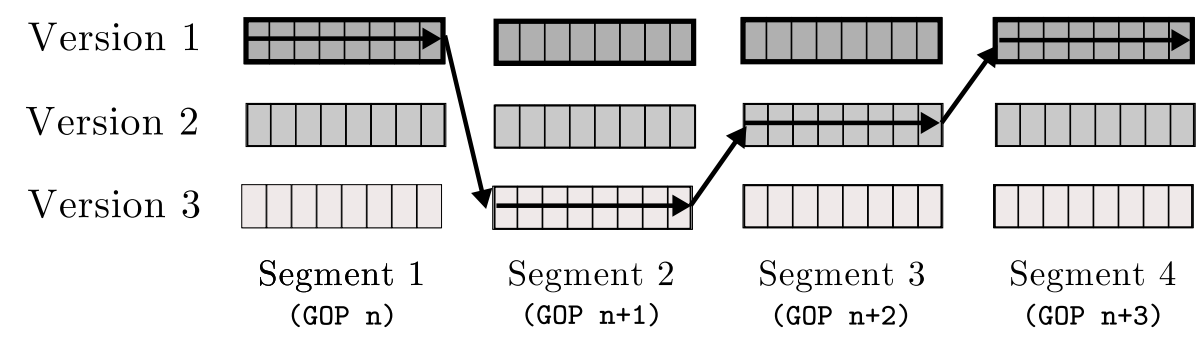

Figure 2. Stream switching technique.

The second type of adaptive video transmission technique is based on the idea of stream switching. This technique encodes the raw video content at several different increasing bit rates, generating $j$ versions of the same content. Figure 2 illustrates an example of this technique, where 3 versions of a same video are generated. Each video version is also divided into segments of the same duration (e.g. using the segmentation based on GOP, Group of Pictures). An algorithm must dynamically choose the video version that matches the available bandwidth of the route. Although this method minimizes processing costs, storage and transmission requirements must be considered because the same video content is encoded $j$ times. Other disadvantage of this method is the coarse granularity since there is only a discrete set of levels. The third type of adaptive video transmission technique employs Scalable Video Coding (H.264/SVC) [2], which has attracted great interest by researchers to improve video streaming over wireless networks [6], [10], [36]. SVC enables the encoding of a video by creating different representations (layers) which can be integrated within a single bit stream. The most basic representation of video is contained in the "base layer", which corresponds to the lowest quality representation. In addition, multiple layers of "enhancement" are generated. These layers provide an increasing of the video quality when they are added to the base layer. The layered scheme of SVC can provide higher robustness during video streaming over networks with continuous fluctuations of the available bandwidth and high packet loss rates. SVC allows the sender to adapt the bit rate of the video traffic by means of adding or removing SVC layers from the video stream based on the estimation of the available bandwidth (Figure 3).

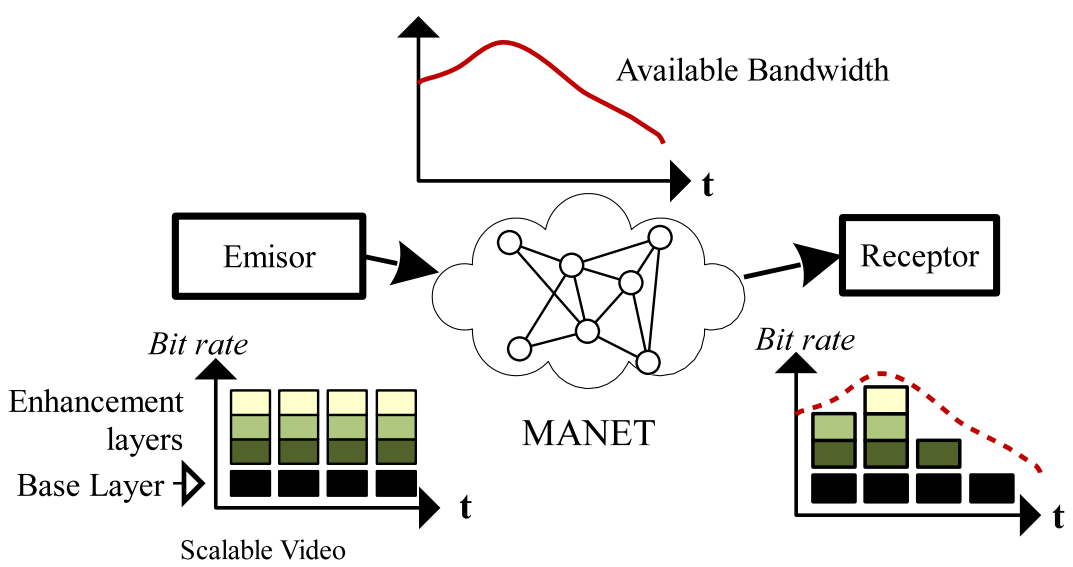

Figure 3. Adaptive scalable video streaming. 
Therefore, in order to dynamically adapt the video stream, it is necessary to have a mechanism to estimate the available bandwidth and report to the source video. This article proposes the use of AQA-AODV protocol (Adaptive QoS-Aware Ad-hoc On-demand Distance Vector) [37] for this purpose. AQA-AODV is an inter-layers (cross-layer) solution that enables: estimating the available bandwidth, information exchange between layers (network, transport and application) and the establishment of routes within the MANET. It also provides feedback to the application layer to report the resources available in the network.

\subsection{Scalable video coding (SVC)}

SVC coding was standardized as an extension of H.264/AVC. A video that has been encoded using H.264/SVC has a layered structure, which corresponds to different levels of quality or different spatial and temporal representations. The main feature of SVC is that it provides three different types of scalability (Figure 4): spatial, temporal and quality (also called SNR scalability, signal-to-noise-ratio).

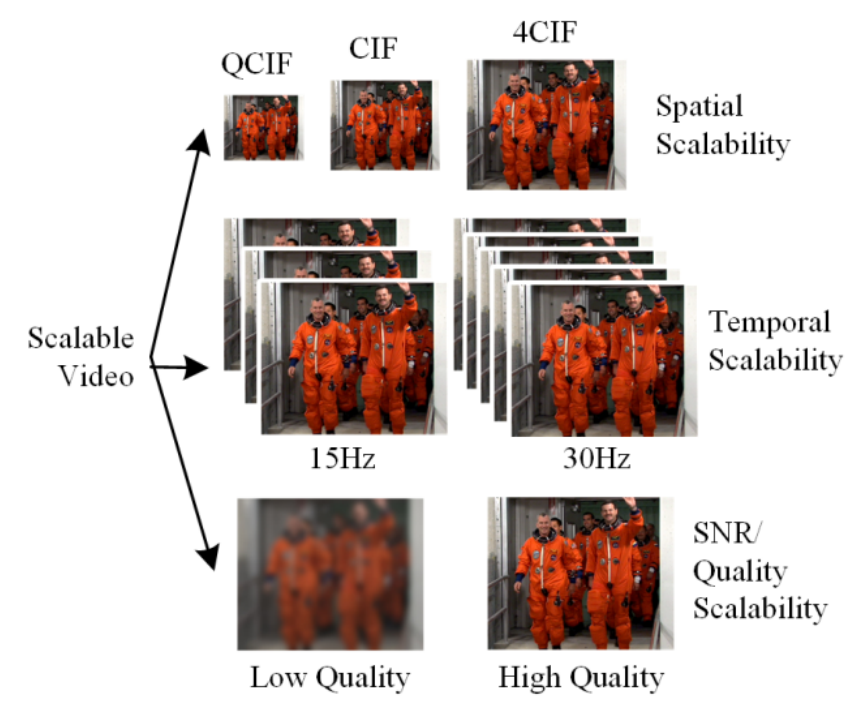

Figure 4. Different types of scalability in H.264/SVC.

Scalability in SVC context means that video information can be retrieved by decoding only part of encoded video. For example, it is possible to decode the video at a lower resolution than the original, decoding only the lowest spatial layer and discarding the other layers. While using temporal scalability, layers improve the frame rate, with spatial scalability, the base layer is encoded at a low spatial resolution (frame size) and enhancement layers give progressively higher spatial resolution (Figure 4). Finally, quality scalability (or SNR) refers to scaling in terms of the level of compression applied to the source video. With quality scalability, the base layer contains a strongly compressed version of each picture, and enhancement layers incorporate more information to increase the SNR value. The H.264/SVC standard supports combined scalability, that is, a SVC video stream can use any combination of the three types of scalability. For instance, Figure 5 shows a SVC stream encoded with three temporal levels $\left\{\mathrm{T}_{0}, \mathrm{~T}_{1}\right.$ and $\left.\mathrm{T}_{2}\right\}$ and two quality levels $\left\{\mathrm{Q}_{0}\right.$ and $\left.\mathrm{Q}_{1}\right\}$. Thus, six scalable layers are generated by combining these levels as is, as Figure 5 shows. Base layer is formed using the lower temporal resolution level $\mathrm{T}_{0}$ and the lower quality level (i.e. frames 0 and 4). An example of enhancement layer may be formed by the temporal level $\mathrm{T}_{2}$ and the quality level $\mathrm{Q}_{1}$. 


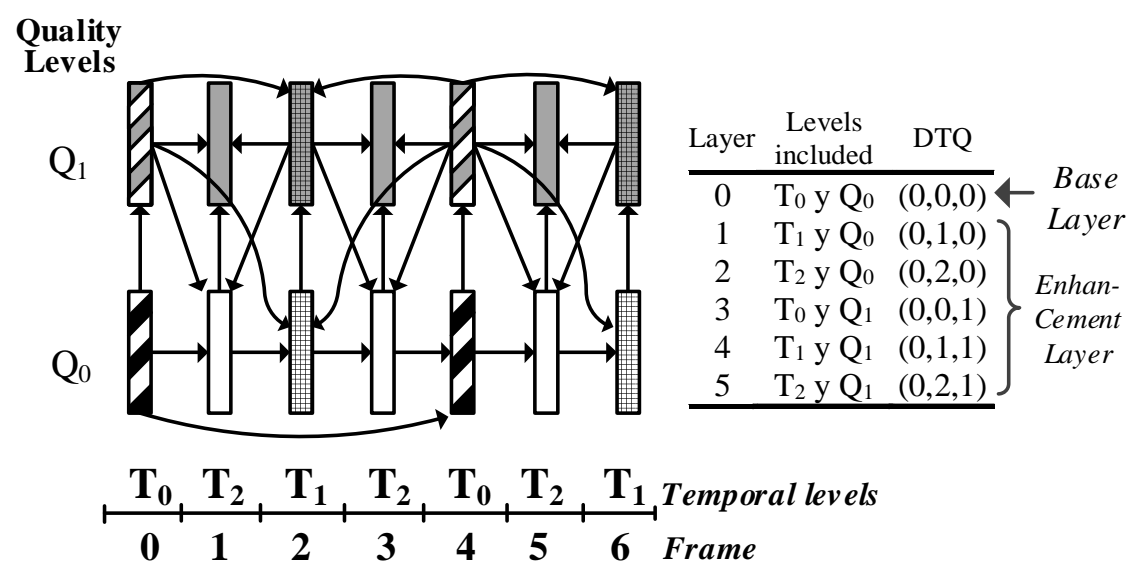

Figure 5. Example of coding structure of a SVC stream with temporal and quality scalability.

In H.264/SVC, the codec is divided in two subsystems: the Video Coding Layer (VCL) and the Network Abstraction Layer (NAL). VCL is responsible for video encoding, and the NAL subsystem formats these data according to the transport layer requirements. The encoded video data are encapsulated into Network Abstraction Layer Units (NALUs). NALUs contain full or partial information of the coded data frames. Each NALU contains three identifiers: dependency identifier (DID), temporal identifier (TID) and quality identifier (QID). These identifiers represent a point in the spatial, temporal and quality scalable dimensions, respectively. The values of DID, TID and QID are also known as (D,T,Q) parameters. For example, a NALU of the base layer should be identified as $(0,0,0)$ and the enhancement layer consisting of the $\mathrm{T}_{2}$ and $\mathrm{Q}_{1}$ levels should be identified as $(0,2,1)$. The inspection of the $(\mathrm{D}, \mathrm{T}, \mathrm{Q})$ values permits to identify the NALUs belonging to a specific enhancement layer. This fact is particularly important since the NALUs may be identified and removed from the SVC encoded video, in order to reduce the bit rate. Despite the elimination of some NALUs, the remaining NALUs could be used to integrate a valid video bit-stream but with a lower visual quality. Nevertheless, the dependencies of the layers would be taken into account. Layers in SVC can be decoded independently but there is a logical dependency between them. This interdependency must be considered in order to obtain a correct decoding of the video. In the example shown in Figure 5, the arrow lines represent dependencies between frames in a combined scalable stream. For instance, the frame 2 of the layer $T_{2} Q_{1}$ depends on the layer $T_{0} Q_{1}$ and the layer $T_{1} Q_{1}$ (which also depends on base layer). Because of these dependencies, discarding a quality layer from a reference frame (e.g. frame 2) affects the quality of dependent frames (e.g. frames 1 and 3).

\subsubsection{Quality Scalability MGS}

The H.264/SVC standard provides three types of quality scalability: Coarse-Grained Scalability (CGS), Medium Grain Scalability (MGS) and Fine Grain scalability (FGS) [20]. In this paper MGS was used for quality scalability as this type of scalability increases the efficiency of coding and enables the ability of changing between layers at any moment in the decoding process. Figure 6 describes the advantage of using MGS over other scalability schemes. With CGS, each enhancement layer is separately estimated. However, this scheme has several disadvantages. For instance, if a part of the enhancement layer is lost, an error will occur during the subsequent sequence estimation (Figure 6a). On the contrary, using the FGS scheme each enhancement layer is predicted from the base layer (Figure 6b). Then, if the packets corresponding to the enhancement layer are lost for some time, the receiver may still decode the enhancement layer from the base layer. The better solution seems to be the MGS scheme, shown in Figure 6c, as there is more flexibility when choosing which of the quality layers can be used for prediction. Furthermore, the concept of reference frames is introduced, which serve to make regular updates in the base layer. In [20] more detailed information about scalable video coding and different scalability schemes can be found. 


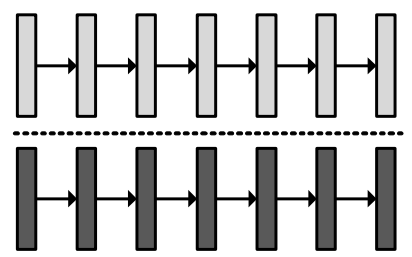

(a) CGS

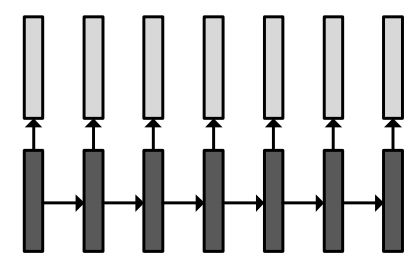

(b) FGS

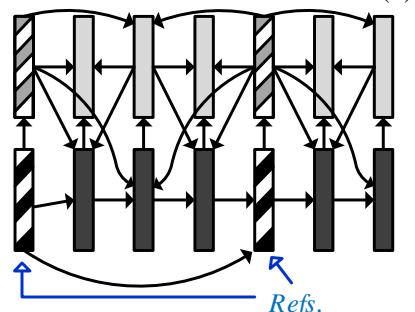

(c) MGS

Figure 6. Quality scalability methods.

\section{DESCRIPTION OF THE SIMULATION FRAMEWORK SVCEVAL-RA}

This section presents a novel simulation framework called SVCEval-RA (SVC Evaluation Platform for RateAdaptive Video), which can be used to carry out performance evaluations of rate-adaptive video streaming using Scalable Video Coding. As mentioned in Section 2, recently there have been developed some software tools to simulate the streaming and evaluation of scalable video as EvalSVC and myEvalSVC. However, these platforms do not contain the necessary algorithms to allow scalable video source to adapt its bit rate, discarding or adding layers that cannot be supported by the network. In contrast to these platforms, SVCEval-RA implements a new procedure in order to adapt the bit rate of the traffic source based on the estimation of the available bandwidth. SVCEval-RA has been integrated to network simulator NS-2, so it can be used along with AQA-AODV protocol to assess the advantages gained in video transmission over ad hoc mobile networks. Figure 7, illustrates the block diagram of the SVCEval-RA evaluation framework. The whole process can be summarized in three main phases, encoding, simulation and decoding. In the encoding phase the original video in YUV format is encoded according to the H.264/SVC standard. Encoding is performed using the JSVM (Joint Scalable Video Model) software [38]. Throughout this phase the JSVM tool encodes a YUV video by configuring all the encoding settings, including the type of scalability to be used, and the number of layers that will compose the output stream. After that, the NALU trace file is produced from the H.264/SVC encoded video using the BitStreamExtractor JSVM tool. This NALU trace file includes a different register for each packet of the NALU sequence. Some of the stored parameters are: the packet size, the $(\mathrm{D}, \mathrm{T}, \mathrm{Q})$ values and the packet type, which are needed during the decoding phase. Before the simulation phase, it is also necessary to generate two additional traces. These traces are generated by SVCEval-RA. On the one hand, the Bit Rate Trace is generated. This Bit Rate Trace file provides information about the bit rate required to transmit each layer of the SVC encoded video. Specifically, it contains: layer id, frame rate (fps), bit rate (Kbps), the (D,T,Q) values and PSNR (dB). Afterward, the NS-2 Traffic Trace is generated by adapting NALU trace file to the appropriated format required by NS-2. 


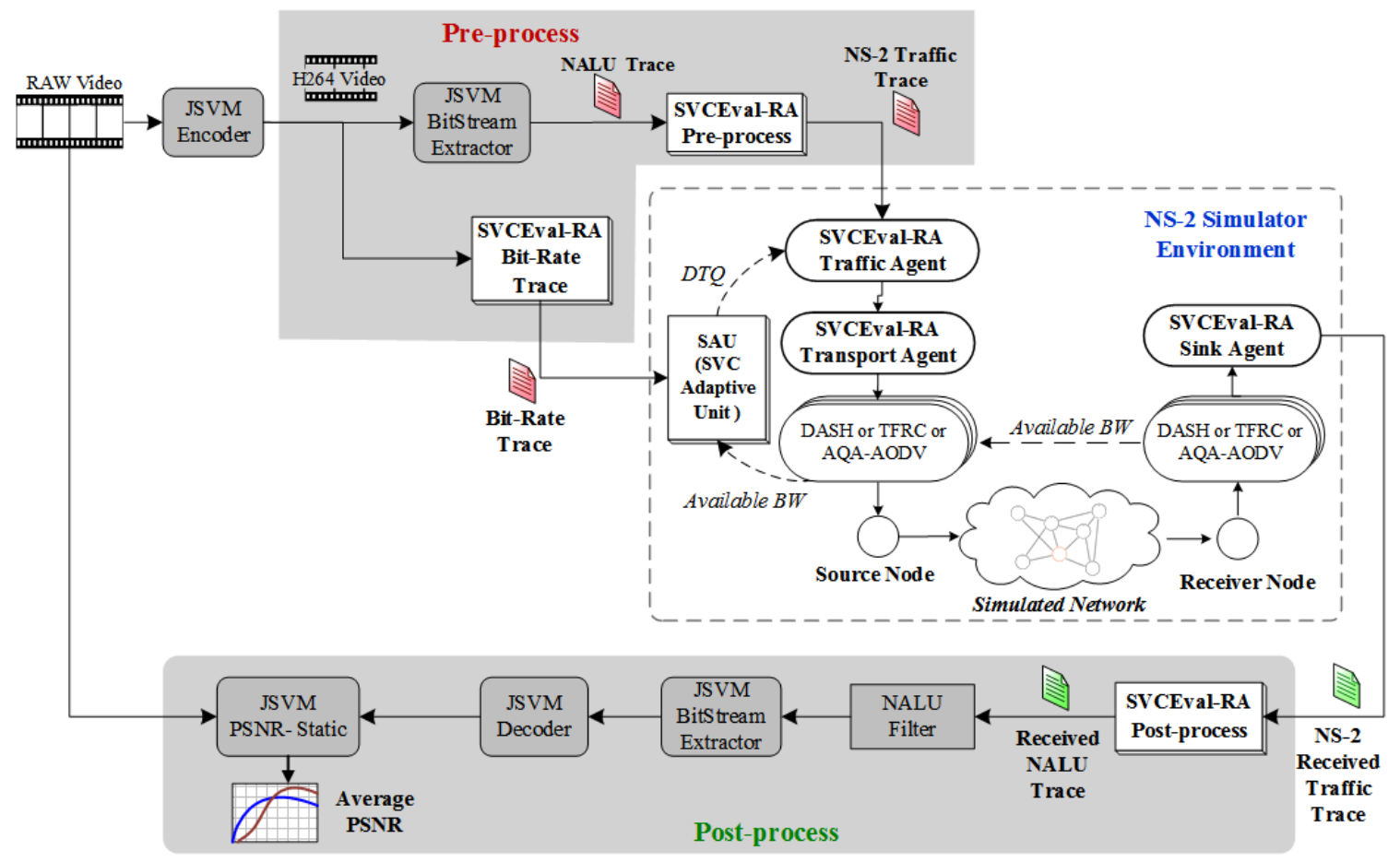

Figure 7. Schematic representation of the SVCEval-RA simulation framework.

During the transmission of video packets over the mobile ad hoc network, AQA-AODV protocol provides the estimated available bandwidth, which is sent to the adaptive unit. In this module, the (D,T,Q) parameters of the highest layer that can be transmitted without exceeding the bandwidth limitation are determined. Therefore, the selected layer has a bit rate lower or equal to the transmission rate supported by the network. From the $(D, T, Q)$ parameters, the software agent SVCEval-RA integrated into the simulator filters the packets corresponding to the aforesaid layer and its lower layers, so that only these are transmitted throughout the network. After simulation, the SVCEval-RA Post-process program first processes the Received Traffic Trace in order to obtain the Received NALU Trace file. Afterward, the Received NALU Trace file is passed to the NALU-Filter tool which: $i$ ) removes NALUs received after the play-out buffer deadline, ii) reorders the NALUs according to the sending order and, iii) removes NALUs with unfulfilled decoding dependencies. The filtered Received NALU Trace file is used to reconstruct the H.264/SVC stream using the BitStreamExtractor and the JSVM Encoder tools. The obtained SVC video can then be decoded to a YUV RAW file by means of the JSVM H264 Decoder Static tool. This final YUV video can be compared with the original YUV video in order to measure the PSNR for evaluating the end-to-end delivered video quality. Furthermore, other performance metrics, such as the packet loss rate and end-to-end delay, can be obtained from the NS-2 Traffic traces generated during network simulation.

\section{AQA-AODV: ROUTING PROTOCOL WITH QoS FOR MOBILE AD HOC NETWORKS}

Besides the development of the simulation framework SVCEval-RA to assess the SVC video streaming over MANETs, we have also implemented a routing protocol that provides mechanisms to achieve certain levels of QoS. This section describes the protocol developed, which has been called Adaptive QoS-Aware Ad-hoc Ondemand Distance Vector (AQA-AODV). This protocol has been developed as an AODV protocol modification. The main modifications influence the pathfinding process and route maintenance strategy of AODV. To implement these changes, new fields were introduced into the packet format used in AODV. For instance, new fields were incorporated into the route request packet (RREQ) to inform the bandwidth required and the session identifier (session ID), used to identify each stream over the network. In addition, the reply packet (RREP) and 
the route error packet (RERR) were modified with new fields. The modified AODV packets in AQA-AODV have been called QRREQ, QRREP and QRRER to emphasize the new role of transport QoS parameters.

The main difference of AQA-AODV with other solutions based on AODV is the adaptive feedback scheme by which the source node may adapt its transmission rate according to the state of the route. Therefore, the nodes along the route must incorporate algorithms that allow them to estimate the resources available (depending on the bandwidth).

The main contributions of AQA-AODV are:

i) An algorithm for estimating the bandwidth available on the network by modifying the format of the message HELLO and creating a new message called HELLOACK.

ii) A "cross-layer" agent that enables information exchange between the application layer and the network layer. This agent informs the network layer of the bandwidth requested by the application layer and the traffic protocol informs the traffic source of the bit rate at which it can transmit.

iii) A new route discovery mechanism (QREEQ and QRREP packets) that take into account the new parameters listed for establishing routes (available bandwidth, session ID and maximum transmission rate).

iv) A new route maintenance and recovery scheme, which incorporates a cache table with all active sessions.

v) Modification of the neighbor cache and the routing table to incorporate the parameter of quality of service (QoS) and the session identifier.

Figure 8 shows the functional diagram of the main modules AQA-AODV. The tree main elements of AQAAODV are: the bandwidth estimation module, the routing module and the route recovery module. The first module performs the estimation of available bandwidth and provides the feedback value to the application layer. Hello packets are used for the estimation. These packets are originally used in AODV in discovering neighboring nodes. With information about the available bandwidth, the traffic source can adjust its transmission rate, as proposed in this article. Furthermore, the routing module receives the route request from the source traffic in which the required transmission rate is indicated, and executes the route search process. The third module is the route recovery module, which is responsible for detecting connectivity faults and re-establish routes to the destination, taking into account the conditions of QoS previously established. A preliminary performance evaluation of AQA-AODV during the transmission of data flows over MANETs was presented in [37].

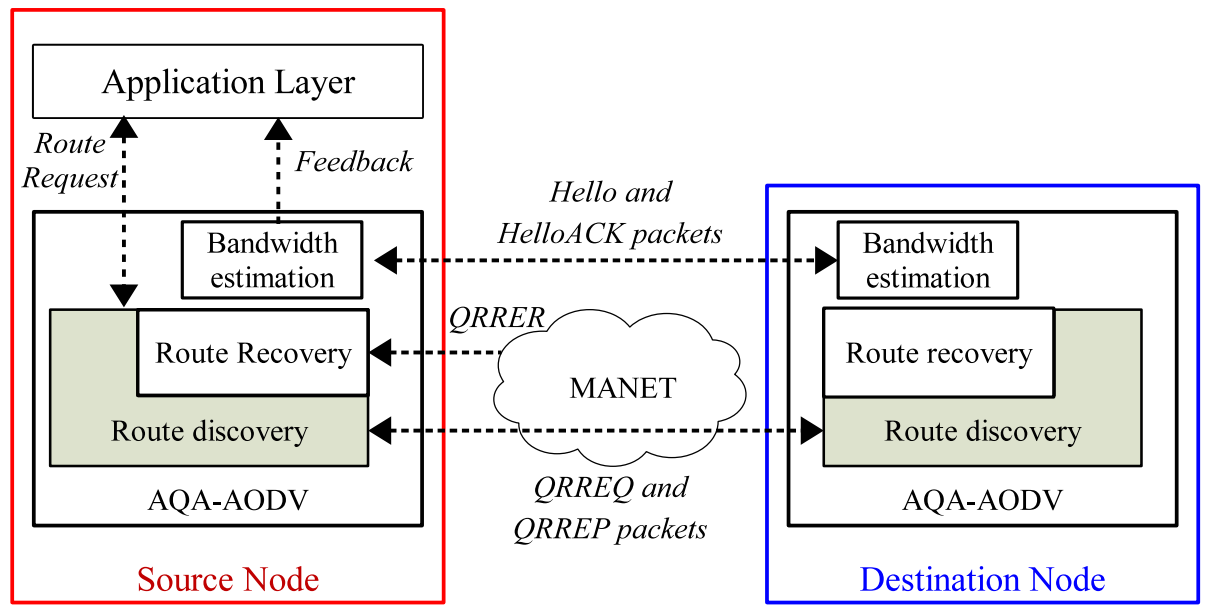

Figure 8. Functional diagram of AQA-AODV.

\section{EVALUATION}

This section describes the parameters used for the simulations. The main objective of the simulations is to evaluate two strategies for scalable video streaming over MANETs: Adaptive SVC and Non-adaptive SVC. The 
adaptive strategy uses AQA-AODV protocol as routing protocol while non-adaptive strategy uses the AODV protocol. This section also aims to show how the integration of our tools (AQA-AODV and SVCEval-RA) contributes to the study of adaptive video streaming over ad hoc mobile networks. The simulations were carried out on two scenarios. The first scenario is an ad hoc wireless network with static nodes and the other scenario is a mobile ad hoc network.

\subsection{Simulation environment}

For the evaluation of SVC traffic on MANETs, the network simulator NS- 2 was used. In Table 1, the parameters of the simulator are described.

Table 1. Simulation Parameters

\begin{tabular}{|c|c|}
\hline Parameter & Value \\
\hline MAC Protocol & $802.11 \mathrm{~b}$ \\
\hline Propagation model & Two Ray Ground \\
\hline Queue type & Drop Tail \\
\hline UDP packet size & 1000 bytes \\
\hline Transmission range & $250 \mathrm{~m}$ \\
\hline Interference range & $550 \mathrm{~m}$ \\
\hline
\end{tabular}

The video used as traffic flow was generated by concatenating three times the sequence "CREW" [39] and obtaining a video of 900 frames with a size of 352x288 pixels. This concatenated sequence was encoded according to the H.264/SVC standard with two types of scalability: temporal and quality (MGS). Encoding was performed using the JSVM software [38]. All the values for the video related parameters are reported in Table 2. The generated SVC stream includes 5 temporal levels, identified as $T_{0}, T_{1}, T_{2}, T_{3}$ and $T_{4}$. Each temporal level has 4 levels of quality $\left(\mathrm{Q}_{0} \mathrm{Q}_{1} \mathrm{Q}_{2}\right.$ and $\left.\mathrm{Q}_{3}\right)$. A total of 20 layers were obtained from the combination of $\mathrm{T}_{\mathrm{i}}$ and $\mathrm{Q}_{\mathrm{j}}$ levels $\left(\mathrm{L}_{0}-\mathrm{L}_{19}\right)$. Depending on the number of transmitted layers, the bit rate varies from $94.4 \mathrm{Kbps}$ (transmitting only layer 0) to $1 \mathrm{Mbps}$ (transmitting all the 20 layers). Table 3 shows the bit rate associated with each layer. These values are accumulative, that is, those bit rates take into account not only the bit rate needed to transmit the data of a specific layer but also include the bit rate needed to transmit the layers that depend on. For example, for the transmission of the layer 3 the total bit rate is $273.3 \mathrm{Kbps}$ (see Table 3). This rate includes layer 3 and the lower layers that depend on (layers 0,1 , and 2).

Table 2. Video Parameters

\begin{tabular}{l|l}
\hline Parameters & Description/Value \\
\hline Original Video file & YUV format \\
Video rate (average) & $36.51 \mathrm{Mbps}$ \\
Video sequence & CREW [39] \\
Resolution & CIF $(352 \times 288)$ \\
Size & 900 frames \\
Frame per second & 30 \\
Duration & $30 \mathrm{~s}$ \\
Encoded video file & H.264/SVC \\
Type of scalability & SNR (MGS) \\
B-Frames & Yes \\
GOP Size & 16 frames \\
\hline
\end{tabular}


Table 3. Description of the SVC Encoded Video Layers

\begin{tabular}{|c|c|c|c|}
\hline Layer ID & TiQi & $\begin{array}{c}\text { Frame rate } \\
(\mathrm{Hz})\end{array}$ & $\begin{array}{c}\text { Cumulative } \\
\text { bitrate (Kbps) }\end{array}$ \\
\hline 0 & T0 Q0 & 1.875 & 94.4 \\
\hline 1 & T1 Q0 & 3.75 & 139.9 \\
\hline 2 & $\mathrm{~T} 2 \mathrm{Q} 0$ & 7.5 & 197.4 \\
\hline 3 & T3 Q0 & 15 & 273.3 \\
\hline 4 & T4 Q0 & 30 & 335.4 \\
\hline 5 & T0 Q1 & 1.875 & 157.0 \\
\hline 6 & T0 Q2 & 1.875 & 197.4 \\
\hline 7 & T0 Q3 & 1.875 & 245.1 \\
\hline 8 & T1 Q1 & 3.75 & 254.9 \\
\hline 9 & T1 Q2 & 3.75 & 314.1 \\
\hline 10 & T1 Q3 & 3.75 & 376.7 \\
\hline 11 & T2 Q1 & 7.5 & 401.5 \\
\hline 12 & T2 Q2 & 7.5 & 487.0 \\
\hline 13 & T2 Q3 & 7.5 & 569.1 \\
\hline 14 & T3 Q1 & 15 & 593.0 \\
\hline 15 & T3 Q2 & 15 & 707.1 \\
\hline 16 & T3 Q3 & 15 & 808.7 \\
\hline 17 & T4 Q1 & 30 & 809.3 \\
\hline 18 & T4 Q2 & 30 & 948.7 \\
\hline 19 & T4 Q3 & 30 & 1066.8 \\
\hline
\end{tabular}

Figure 9 gives a graphical description of the bit rates obtained according to the temporal levels and the MGS layers. The labels on the bars indicate the layer id assigned by the SVC encoder. Moreover, a rate-distortion analysis in terms of average Y-PSNR (PSNR for the luminance component in the YUV colour space) versus average bit rate was computed off-line (see Figure 10).

The computation of the Y-PSNR curves were performed by stripping out the layers, measuring the average bit rate, decoding the resulting video, and computing the average Y-PSNR. Each of these curves represents a temporal layer and each point corresponds to a MGS layer (from $Q_{0}$ to $Q_{3}$ ). This figure describes the increase in the video quality (in terms of Y-PSNR) depending on the number of quality and temporal layers that make up the video. In the two simulated scenarios, the percentage of lost packets, the luminance PSNR (Y-PSNR) and the decoded frame rate were calculated. This latter metric is the number of successfully decoded frames divided by the total number of frames. 


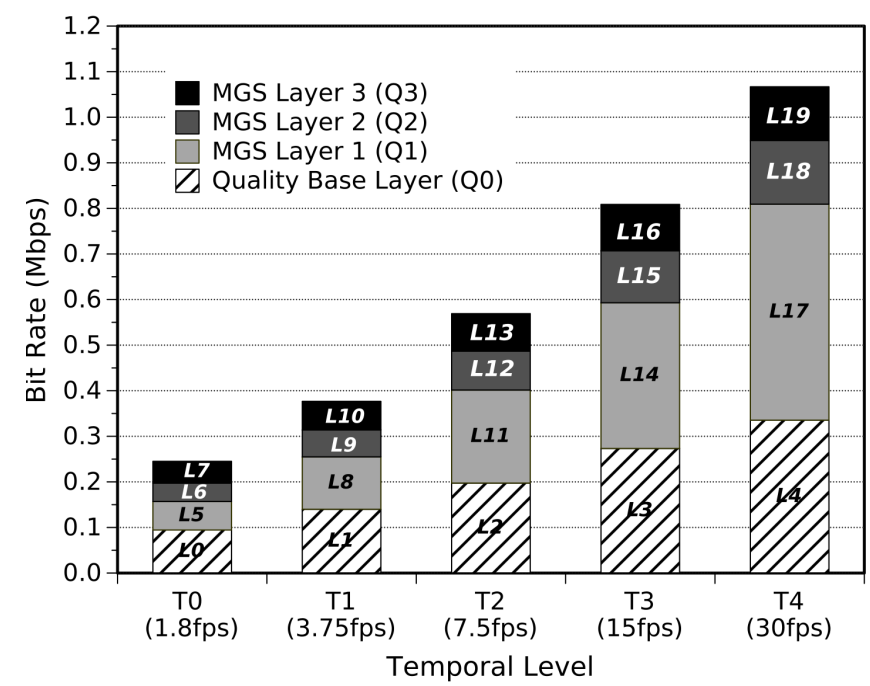

Figure 9. Description: SVC layers contained in the video stream.

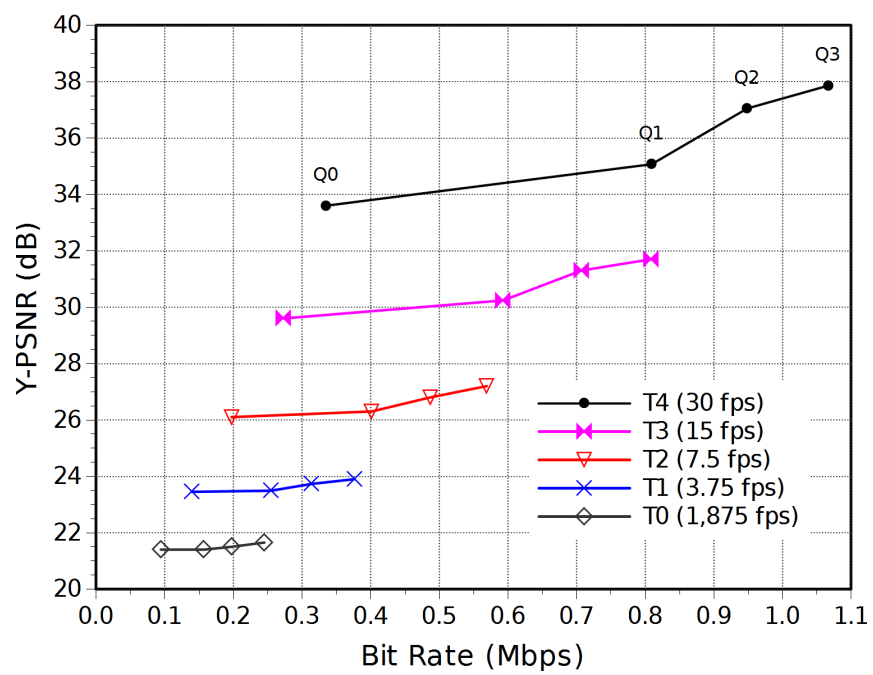

Figure 10. Rate-distortion analysis of the SVC video stream.

\section{RESULTS}

In this section we present simulation results of our proposal in two scenarios: a static topology and a mobile topology. Specifically, we evaluated the transmission of SVC streams by means of the rate-adaptive strategy versus a non-adaptive scheme.

\subsection{Scenario 1: Static linear topology with variable length}

Scenario 1 is a wireless network whose nodes remain static and located in a linear topology (Figure 11). Node 1 is the video source and the last node in the chain is the destination node. In this scenario, the number of nodes in the network increases from 2-15 nodes. The objective is to evaluate the adaptation of the bit rate of the traffic source according to the number of nodes. Figure 12 shows the results of the simulation, as described below: the variation of the available bandwidth estimated by AQA-AODV as a function on the number of nodes in the network is described in Figure 12a; Figure 12b shows the percentage of lost packets, Figure 12c describes the variation of the decoded frame rate, and Figure $12 \mathrm{~d}$ depicts the received video quality in terms of Y-PSNR. Each figure shows the results obtained for both adaptive SVC streaming and Non-adaptive streaming. These curves vary depending on the number of nodes making up the route between the source node and the receiver.

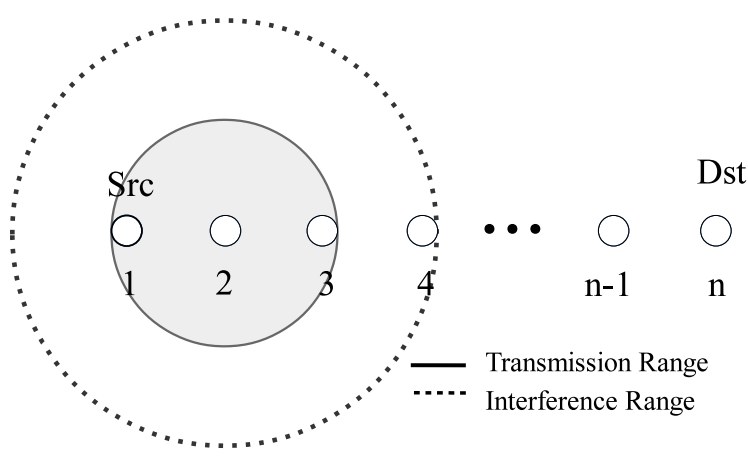

Figure 11. Scenario 1: Static linear topology. 

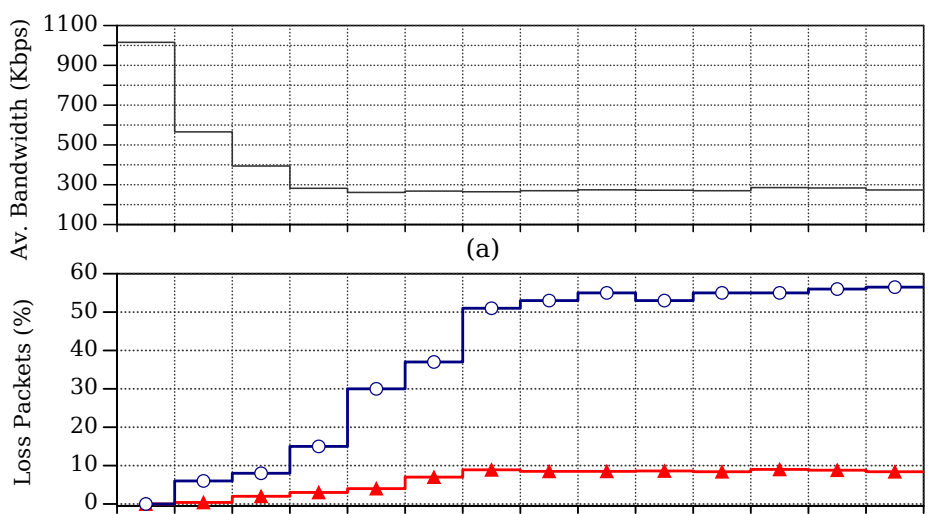

(b)
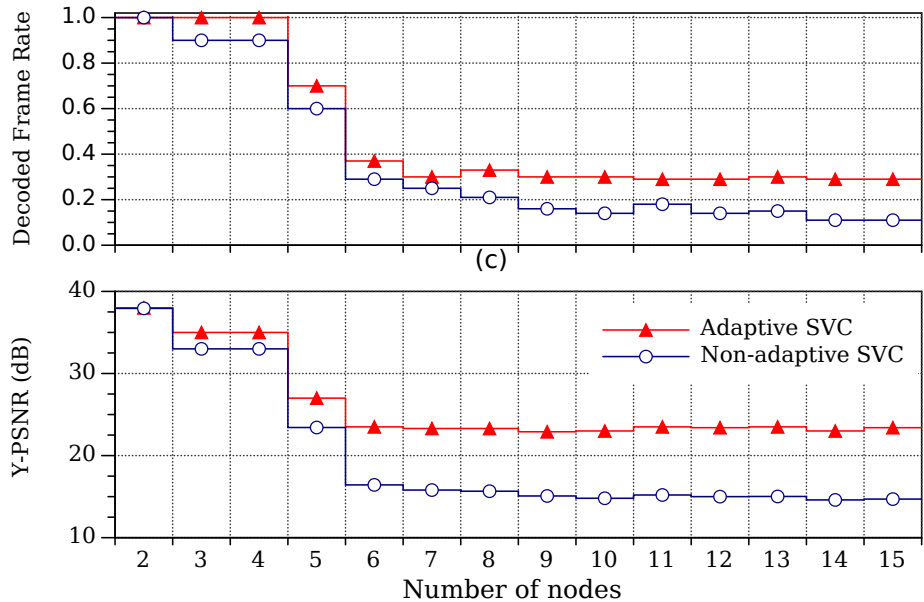

(d)

Figure 12. Results of Scenario 1: (a) available bandwidth, (b) lost packets, (c) decoded frame rate, (d) PSNR, depending on the path length.

As can be seen in Figure 12a, when the path between the source and the receiver has 2 intermediate nodes (i.e. 1 hop), all SVC layers of video can be transmitted. This is because the bandwidth available on the network is sufficient to support the bit rate required to transmit the highest layer (1066 Kbps for layer 19) and the lower layers. Therefore, all frames are successfully decoded (decoded frame rate equal to 1) and the video quality received corresponds to the highest quality that can be achieved (38dB). However, the available bandwidth decreases as the number of nodes on the route increases so that it is no longer possible to transmit all layers and the traffic source should adjust its bit rate. Thus, when the route between source and receiver has 3 or 4 nodes, the video source can transmit layer $4\left(\mathrm{~T}_{4} \mathrm{Q}_{0}\right)$ and lower layers at most. Then, $100 \%$ of frames are successfully decoded, but with lower quality (Y-PSNR of $35 \mathrm{~dB}$ ). However, when the video source does not adapt its bit rate (No-Adaptive SVC curve) some packets are lost (approximately 10\%). Nevertheless, for this percentage of loss, video quality is hardly affected mainly due to the robustness of the SVC coding. When the route consists of more than 5 nodes, the available bandwidth drops dramatically to $280 \mathrm{Kbps}$. With this bandwidth, only Layer $3\left(\mathrm{~T}_{3} \mathrm{Q}_{0}\right)$ and its lower layers could be transmitted, according to the data in Table 3. In Figure 12b, it can be seen that the strategy of adaptive SVC allows the source to adjust its transmission rate to avoid network congestion.

Accordingly, the percentage of lost packets does not exceed 10\% and PSNR of approximately $23 \mathrm{~dB}$ is obtained (Figure 12d). By contrast, with the strategy of using non-adaptive SVC streaming, the percentage of lost packets reaches 50\% (about 5 times more than using adaptive SVC). Hence, the decoded frame rate decreases to 0.1 and the video quality received decreases to $14 \mathrm{~dB}$ (about $9 \mathrm{~dB}$ less than adaptive SVC) in the worst case.

\subsection{Scenario 2: Mobile topology}

The second scenario is a cellular network of 30 nodes in an area of $1000 \times 1000 \mathrm{~m}$. The nodes move to randomly destination at a rate between 0.1 and $2 \mathrm{~m} / \mathrm{s}$ using Random Waypoint Model. When they reach destination, nodes 
pause and then move to another destination. The duration of the pause varies between 0-80 seconds in simulation. Therefore, the relative speed of nodes also varies. A pause time of 0 seconds is the worst case because the nodes are continuously moving. The video stream is transmitted between two nodes (source and destination node) randomly selected. At the beginning of the simulation, the traffic source starts to transmit at the maximum possible speed, i.e. $1 \mathrm{Mbps}$ (maximum bit rate to transmit all SVC layers, see Table 3). In addition to the video stream, $3 \mathrm{CBR}$ streams of $10 \mathrm{Kbps}$ are also transmitted over the network as background traffic using UDP.

The purpose of this stage is to evaluate the transmission of the two SVC schemes on mobile ad hoc networks and measure the impact of mobility over them.

Figure 13 shows the results for the mobile scenario. Regarding packet loss (Figure 13a), the adaptive streaming scheme allows SVC to avoid network congestion and packet loss percentage does not exceed $11 \%$, both for high and low mobility scenarios. Regarding video quality, Figure 13b shows that, for high mobility, both transmission strategies have a significant decrease in the number of successfully decoded frames. For adaptive SVC, this is mainly due to the extraction of temporal layers from the SVC stream, carried out by the traffic source in order to adapt the transmission rate to the available bandwidth. On the contrary, the low rate of decoded frames using Non-adaptive SVC is due to both the high percentage of packets lost and the elimination of NALUs received with excessive delay. Finally, a significant improvement is observed in received video quality using Adaptive SVC against Non-adaptive SVC technique (Figure 13c). For the worst case (pause time equal to 0 seconds), there is an improvement in PSNR of $9 \mathrm{~dB}$, whereas with low mobility an improvement from $9 \mathrm{~dB}$ to $13 \mathrm{~dB}$ is obtained.

Although not to the same extent, the mobility of nodes affects both SVC video streaming strategies. However, the adaptive scheme clearly provides better results regarding the received video quality. In addition, it also helps avoid or reduce congestion in the network, minimizing the impact over other traffic streams that would be transmitted over the network.

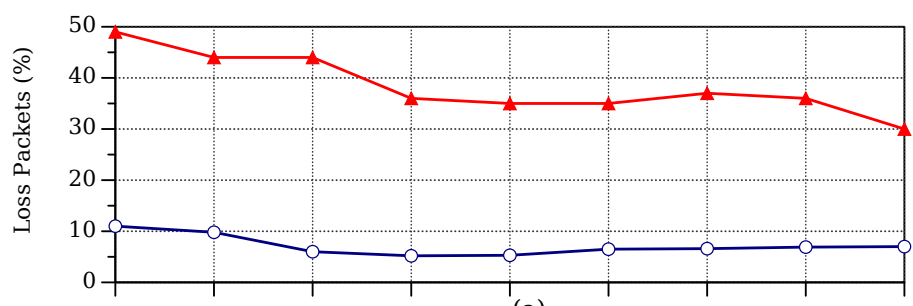

(a)
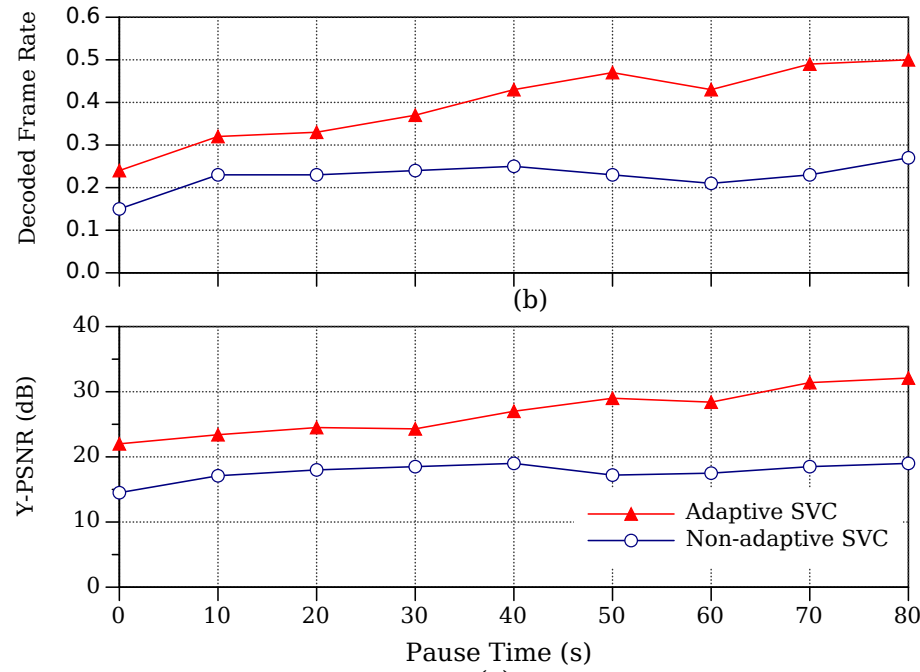

(c)

Figure 13. Results of Scenario Mobile: (a) lost packets, (b) decoded frame rate and (c) PSNR as a function of pause time. 


\section{TESTBED EVALUATION AND RESULTS}

\subsection{Ad hoc node implementation}

Figure 14 shows the functional diagram for the Ad Hoc nodes implemented on the Raspberry Pi platform. The Ad Hoc mode configuration is performed on the files described in the block Network/Synchronization; additionally an NTP client was configured to synchronize the nodes during startup. The choice of the wireless card was carried out taking into account the prior experimentation with several models; the main constraints were the compatibility of drivers with the development platform and the operation of the cards in a real ad hoc communication mode. Taking into account the results of the tests, Awuso36nh card (Linux driver rt2800/chipset RT3070) [40] was selected. The nodes are powered by power bank of $10000 \mathrm{mAh}$, allowing easy deployment for outdoor tests. At user level several free distribution tools have been installed, specifically the Mp4trace tool available in the package Evalvid, the traffic generator Iperf, Tcpdump and Tcpstat for capture and analysis of traffic. The transmitter node stores the set of videos which will be used in the test.

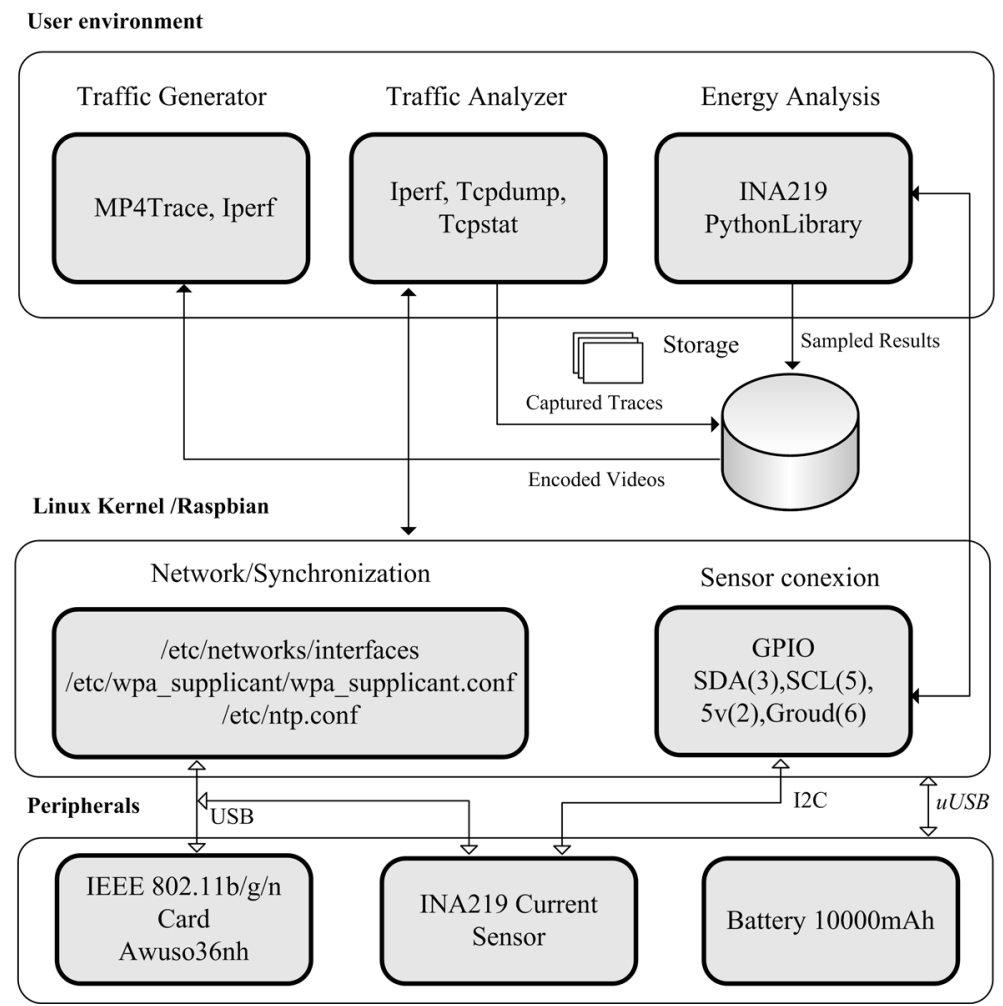

Figure 14. Functional diagram of: Ad hoc node implemented on the development platform Rapberry Pi B+.

Additionally the intensity sensor INA219 [41], has been incorporated into the transmitting node, in order to assess the level of average power consumption demanded by the wireless card. The sensor is handled by a set of Python libraries developed by Adafruit [42]. The connection to the platform is via the I2C (Inter - Integrated Circuit) bus in the GPIO pins (General Purpose Input / Output). Figure 15 shows the ad hoc node implemented. Specifically, Figure 15 (a) depicts the components and Figure 15 (b) presents the setup for real experiments.

For experimental evaluation, we have implemented a total of 10 nodes. The aim of the testbed is to evaluate the adaptive transmission mechanism implementing scenario 1, corresponding to a chain of static nodes located in a linear topology. The AQA-AODV protocol has not been implemented over our platforms yet, therefore in order to select the adaptive flows, we carried out preliminary experiments to measure available bandwidth in the links. Specifically, it was necessary to design and set up multiple tests in order to establish the effective transmission distance as well as characterize the throughput degradation during the transmission in the chain of nodes on the 
real environment. Afterwards, we made the experimental evaluation with the proper flow of video for each bandwidth value. The experiments performed and the results obtained are described below.

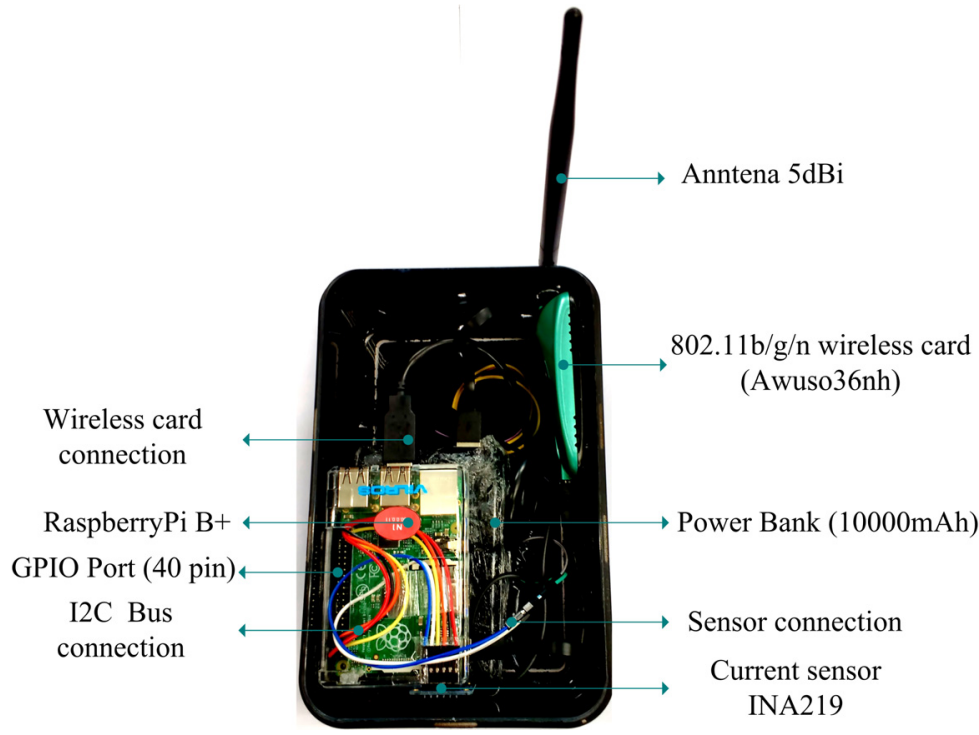

(a)

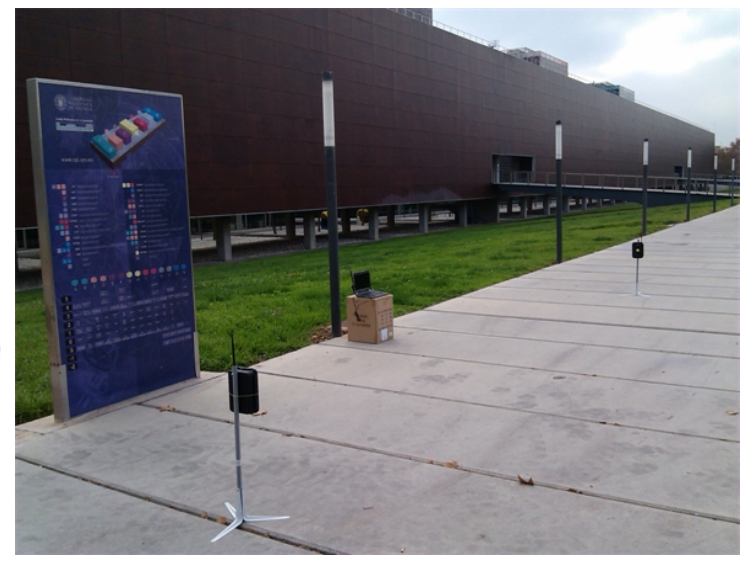

(b)

Figure 15. Ad hoc node implemented: (a) Node components, (b) Setup in a real environment.

\subsection{Experiment 1: Effective Transmission Range}

In this first step, the maximum transmission distance of the wireless card on the real environment was characterized. Tx and Rx nodes were located at different distances in increments of $1 \mathrm{~m}$ and in each case the percentage of packets obtained in reception was evaluated. Data capture is performed at each node running tcpdump, whereas mp4trace was used to transmit video packets. The video used as traffic source corresponds to 1 minute from the popular video sequence Big Buck Bunny [43]. Table 4, describes the traffic parameters set for this specific case and the settings of the wireless card, which are maintained throughout all the experiments.

Table 4. Wireless card configuration and traffic characteristics

\begin{tabular}{l|l}
\hline Parameter & Description/Value \\
\hline MacProtocol & $802.11 \mathrm{~g}$ \\
Tx Power & $0 \mathrm{dBm}$ \\
Data Rate & $54 \mathrm{Mbps}$ \\
Rx Sensitivity & $-76 \mathrm{dBm}$ \\
Anntena Gain & $5 \mathrm{dBi}$ \\
Video sequence & $300 \mathrm{kbps}$ Big Buck Bunny [43] (Coded BitRate mode). \\
\hline
\end{tabular}

Figure 16 presents the results of the experiment. As shown, reception rate remains constant (100\%) up to a distance of $30 \mathrm{~m}$, and then fluctuations in the rate appear, and increase significantly with distance. The variations obtained indicate that beyond the range of $30 \mathrm{~m}$, configuration parameters of the wireless card do not assure a power level that reaches the reception threshold. Therefore, this area is highly susceptible to data loss. Finally, the reception rate is reduced to zero at $59 \mathrm{~m}$. Thus, we select $30 \mathrm{~m}$ as the transmission range in order to set up the 
lineal topology scenario. Moreover, in regard to PSNR, the behavior is coherent to the previous result, with steady and fluctuating zones. PSNR variations depend mainly on the type of frame that lost packets belong to.

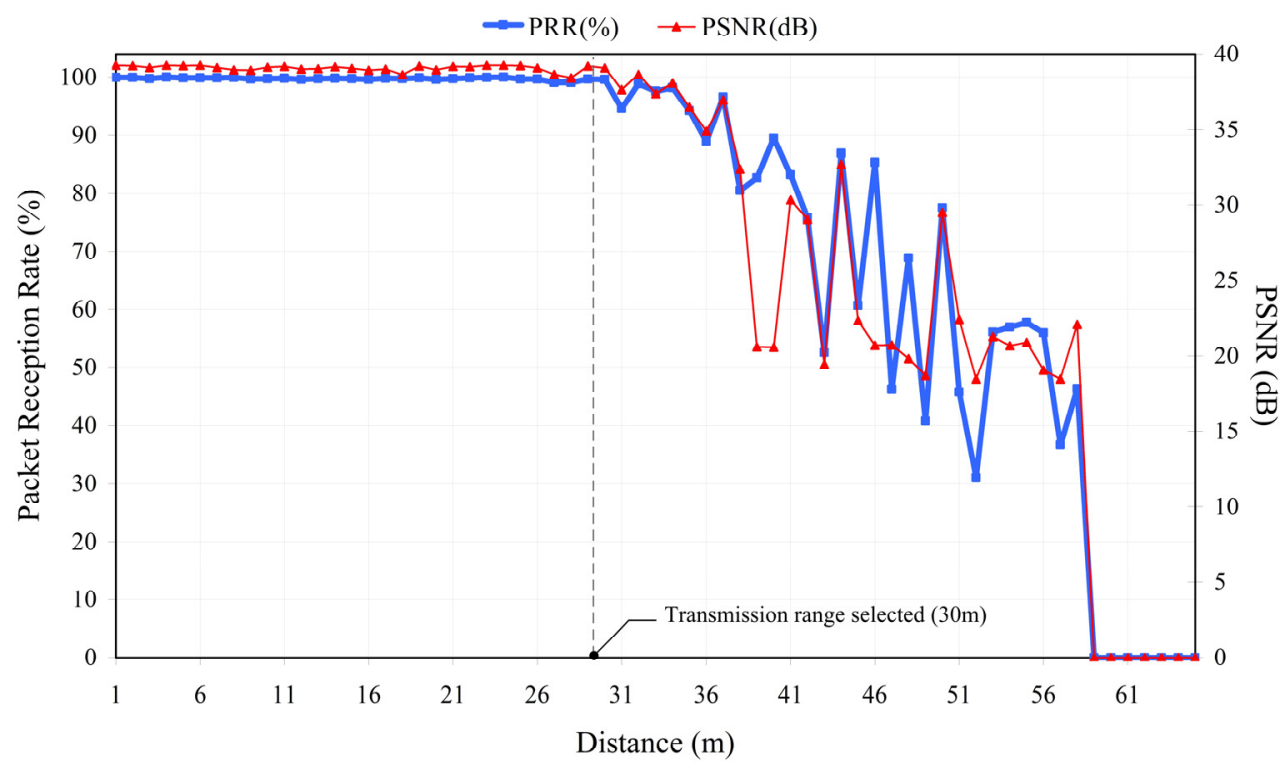

Figure 16. Experiment results: Transmission Range and PSNR.

\subsection{Experiment 2: Throughput Characterization}

This experiment was implemented in order to get a characterization of the actual throughput in the linear topology with increasing number of nodes. The devices were placed at intervals of $30 \mathrm{~m}$ in order to reduce the interference between two hop neighbors. The length of the node chain varied from 2 to 9 nodes. Figure 17 shows a diagram about the implementation of the linear topology.

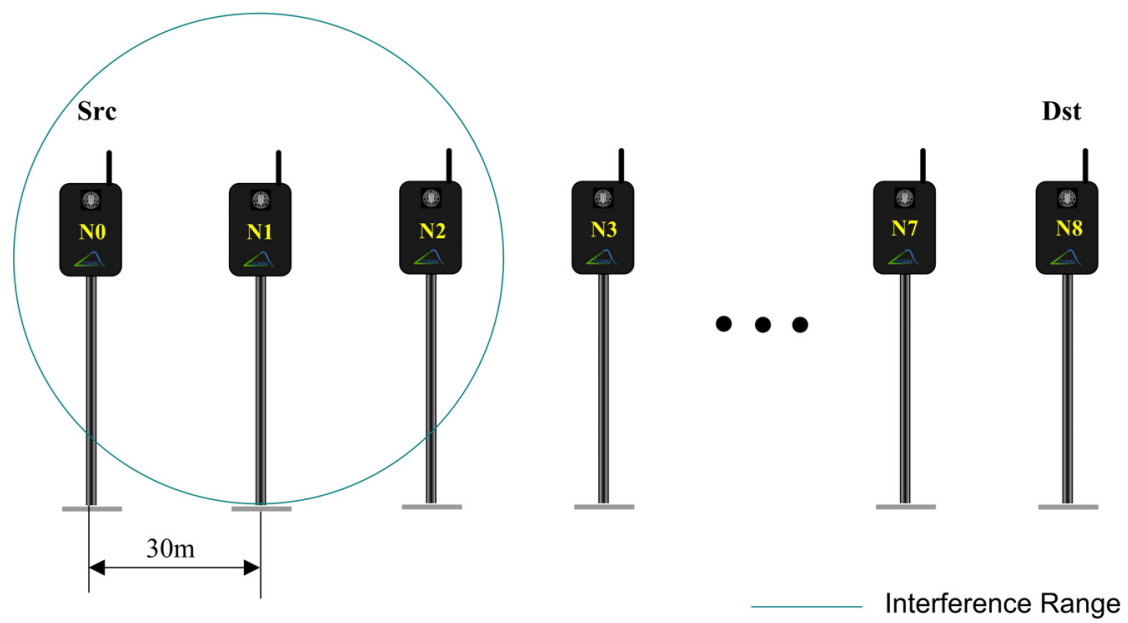

Figure 17. Descriptive diagram for the experiment to determine the effective throughput in the static linear topology.

For the tests, a CBR traffic generator was employed (Iperf tool) and packets were captured using tcpdump. The transmission rate varies from $100 \mathrm{Kbps}$ to $54 \mathrm{Mbps}$. Routing tables were maintained by the routing protocol installed in each Raspberry Pi. Figure 18 presents a description about the throughput obtained in the receiver node 
in comparison with the offered load. The confidence intervals showed were stated at the $95 \%$ confidence level. As it can be seen, a linear trend is maintained for the different topology lengths within an interval (aprox. 13Mbps, $5 \mathrm{Mbps}$, 4Mbps, 3Mbps for 2, 3, 4 and 5 nodes, respectively). The degradation obtained after the linear behavior is due to the shared medium simultaneous access, which limit the transmission opportunity. Specifically, as the number of nodes increases, the contention for the channel is intensified and, therefore, the opportunity to transmit data decreases.

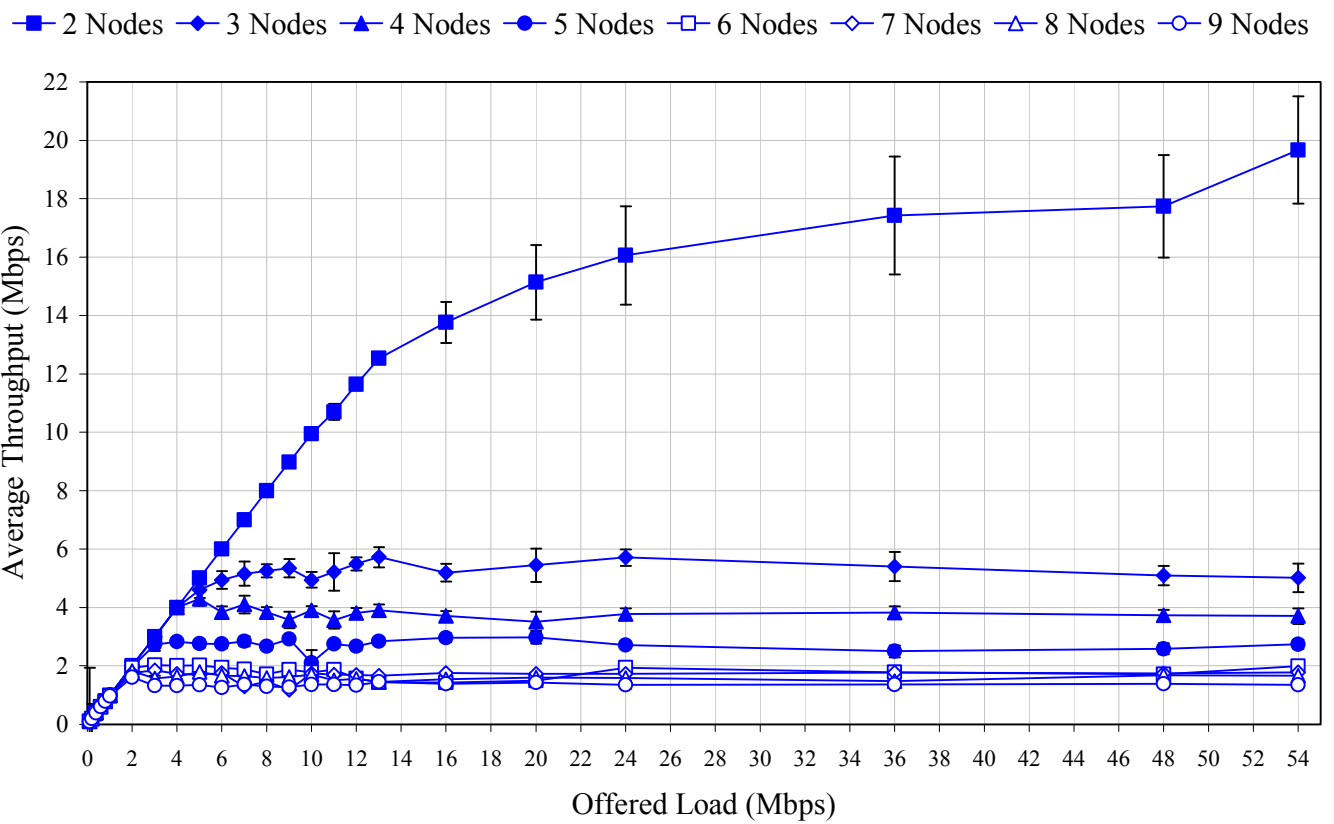

Figure 18. Comparison between the average throughput and the offered load in the static linear topology at the Rx node.

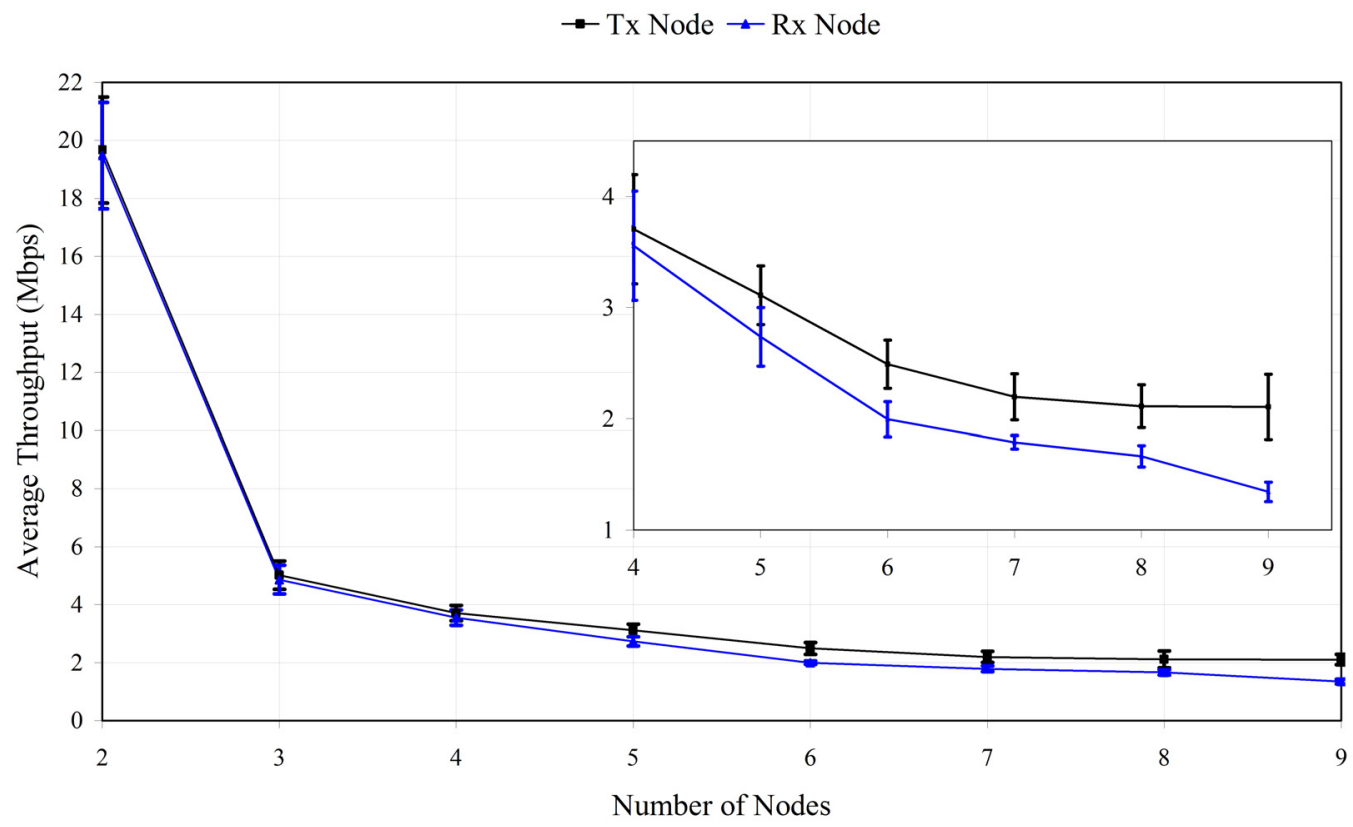

Figure 19. Throughput degradation obtained on the nodes Tx and Rx.

Finally, in order to characterize the throughput degradation from the perspective of both the transmitter and the receiver, results for the offered load of 54Mbps were employed (i.e the highest data rates reached). Figure 19 
shows the average throughput (95\% confidence level) depending on the chain length. In the case of two nodes, the average values of throughput are practically the same in Tx and Rx node. However, as the number of nodes increases, the throughput decreases in the reception side. Such behavior is due to the different levels of congestion in the nodes according to their location in the topology. Particularly, the contention for the channel access is more intense at the ending nodes, causing the data rate reduction obtained in the Rx node.

\subsection{Experiment 3: Evaluation of the adaptive transmission}

This experiment was carried out in order to assess the performance of the adaptive mechanism proposed in this paper. Unlike in the simulations, the standard $802.11 \mathrm{~g}$ was used instead due to the physical layout and the capabilities of the wireless card. In this sense, a new set of video qualities with a higher bitrate is generated, so that the channel bandwidth can be totally used. The sequence "CREW" (300 frames) was employed and layer features are described in Table 5.

Table 5. Description of the video layer for the experimental tests

\begin{tabular}{|c|c|c|c|c|c|}
\hline Layer ID & TiQi & Frame Rate (Hz) & Av. BitRate(Mbps) & Max.BitRate(Mbps) & PSNR (dB) \\
\hline 0 & T0Q0 & 1.875 & 0.052 & 0.086 & 18.10 \\
\hline 1 & T1Q0 & 3.75 & 0.097 & 0.184 & 18.06 \\
\hline 2 & T2Q0 & 7.5 & 0.160 & 0.351 & 18.04 \\
\hline 3 & T3Q0 & 15 & 0.252 & 0.745 & 18.05 \\
\hline 4 & T4Q0 & 30 & 0.382 & 1.608 & 18.59 \\
\hline 5 & T0Q1 & 1.875 & 0.105 & 0.171 & 18.53 \\
\hline 6 & T0Q2 & 1.875 & 0.205 & 0.316 & 18.51 \\
\hline 7 & T0Q3 & 1.875 & 0.378 & 0.531 & 18.51 \\
\hline 8 & T1Q1 & 3.75 & 0.203 & 0.351 & 19.56 \\
\hline 9 & T1Q2 & 3.75 & 0.408 & 0.632 & 19.49 \\
\hline 10 & T1Q3 & 3.75 & 0.765 & 1.056 & 19.46 \\
\hline 11 & T2Q1 & 7.5 & 0.347 & 0.699 & 19.46 \\
\hline 12 & T2Q2 & 7.5 & 0.724 & 1.273 & 21.13 \\
\hline 13 & T2Q3 & 7.5 & 1.423 & 2.156 & 21.06 \\
\hline 14 & T3Q1 & 15 & 0.554 & 1.426 & 21.03 \\
\hline 15 & T3Q2 & 15 & 1.202 & 2.590 & 21.04 \\
\hline 16 & T3Q3 & 15 & 2.523 & 4.294 & 34.01 \\
\hline 17 & T4Q1 & 30 & 0.848 & 3.082 & 37.67 \\
\hline 18 & T4Q2 & 30 & 1.904 & 5.456 & 41.57 \\
\hline 19 & T4Q3 & 30 & 4.335 & 8.904 & 46.02 \\
\hline
\end{tabular}

On the one hand, layer 19, which is the layer with higher bitrate because it includes every lower layer, is used as the non-adaptive sequence. On the other hand, the layer that best fits the available bandwidth has been selected depending on the reference curve obtained (Rx node) from the previous experiments and the average bitrate of each layer (Figure 19). The video sequence was transmitted using the adaptive and the non-adaptive schemes for the different chain lengths described previously. However, after multiple iterations, high packet losses were obtained. Even the adaptive scheme showed higher packet losses than expected. This is due to the bitrate variation inherent to the video encoding nature and the kind and size of encoded frames. In this sense, [44] presents an analysis about the instantaneous throughput that a video burst generates and suggests a traffic load analysis taking into account the frame rate. Consequently, the video sequence was profiled in order to determine the size of the packet bursts, included in Table 5. Figure 20 shows an example of the layer 19 profile. As it can be observed, there are a number of frames that generate a throughput higher than the average bitrate. Therefore, the layer for adaptation should not be selected depending on the average bitrate but attending to the maximum bitrate. 


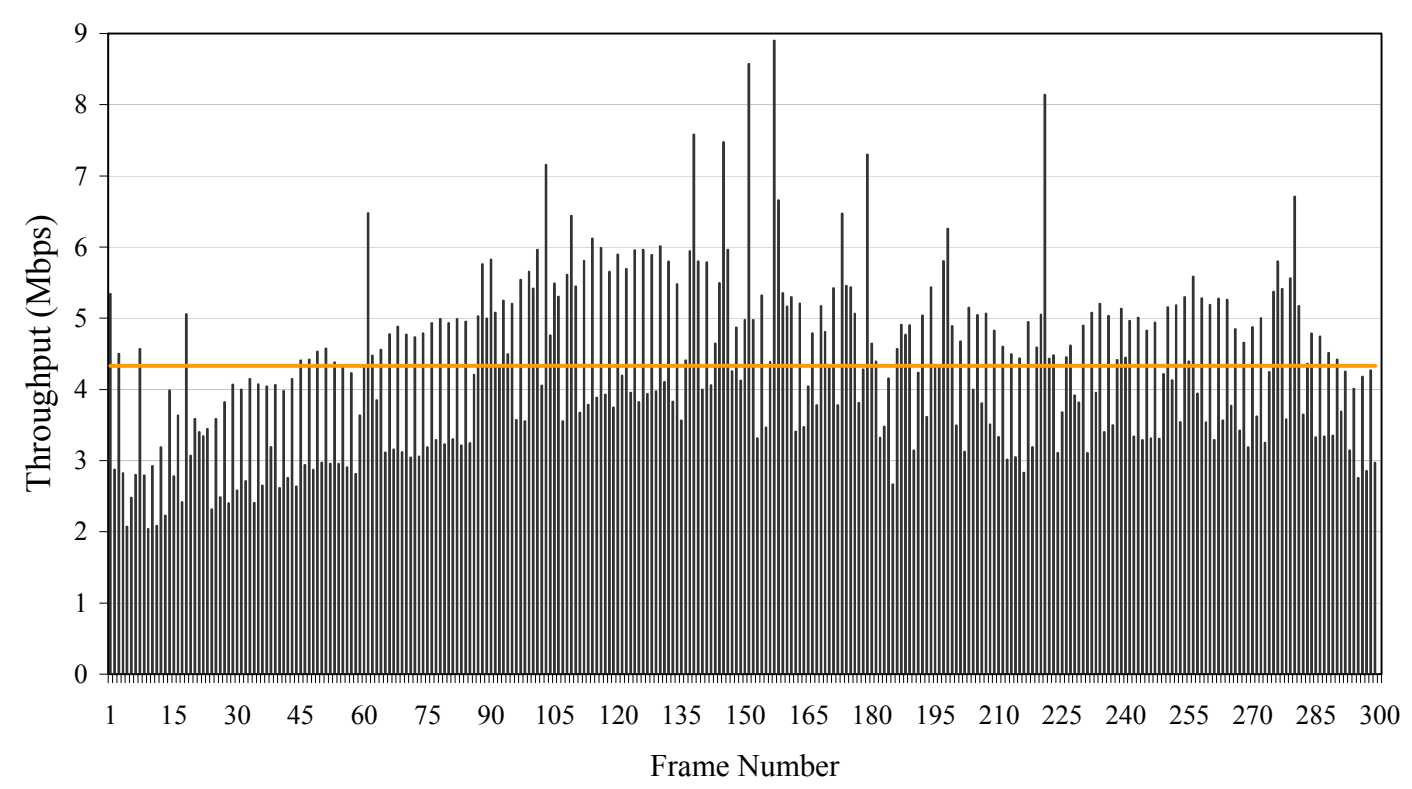

Figure 20. Video profile characterization: Layer 19 (30fps).

In order to explain and analyze this behavior thoroughly, the results for the chain of 6 nodes have been detailed and explained below. As aforementioned, layer 19 has the higher bitrate and is used as the non-adaptive sequence. Layers 14, 12 and 0 were used for evaluating packet losses, in case the adaptive mechanism behaves with different conservative shades. Packet loss depending on the used layer can be shown in Figure 21. Results show that for the adapted bitrate sequence, packet loss is lower (11\% and $15 \%$ for layers 14 and 12, respectively) compared to non-adaptive transmission (23\%). However, it is worth noting the wide confidence intervals, $(95 \%$ confidence level) meaning high variation among iterations. Even with the lower quality layer, losses around $2 \%$ are obtained. Therefore, real environments have proven to be unstable and unpredictable. However, with the adaptive scheme, nodes send fewer packets, causing less congestion to the network. In this sense, an additional experiment has been carried out with the aim of analyzing the power consumption of nodes depending on the throughput.

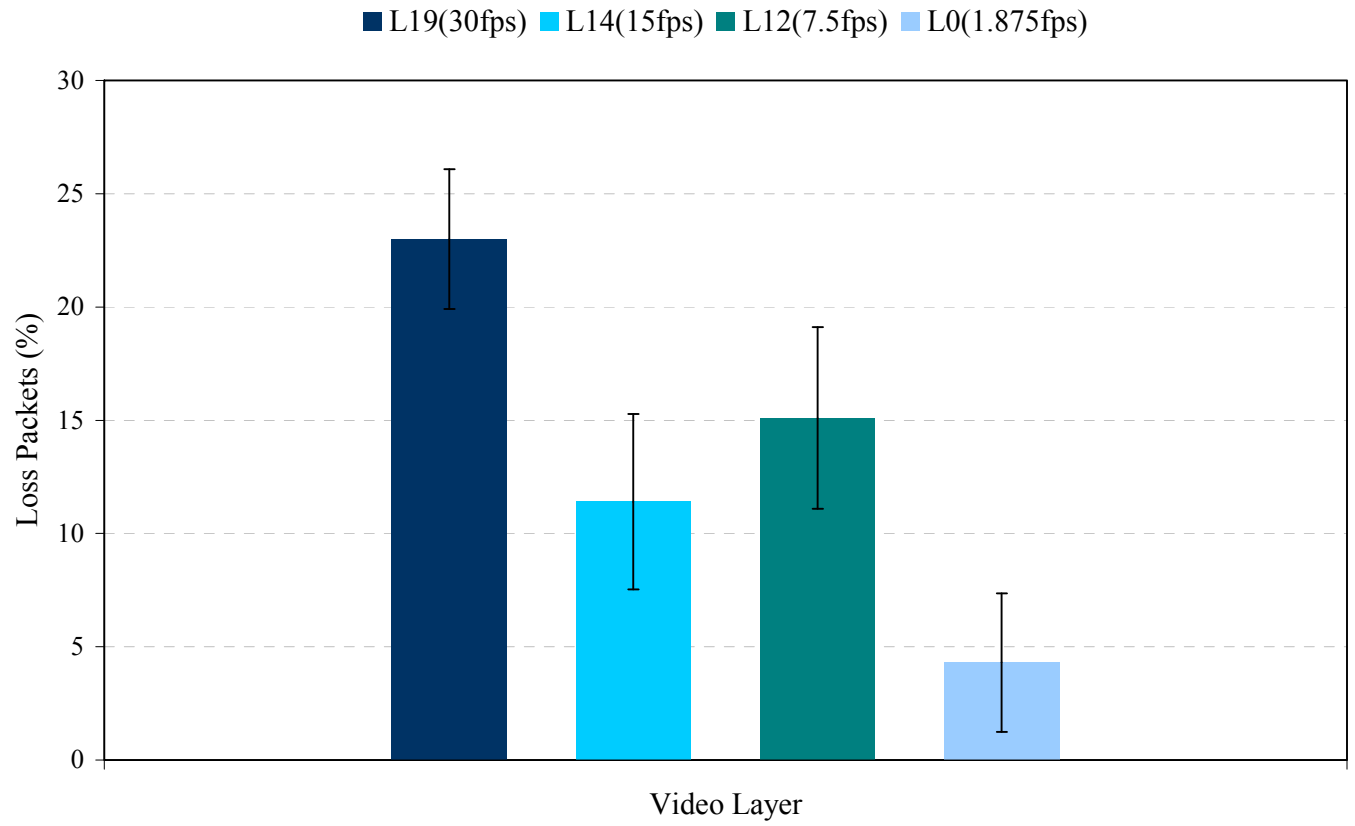

Figure 21. Experiment results: Loss packets(\%) obtained for the lineal topology of 6 nodes, with different traffic flows. 


\subsection{Experiment 4: Evaluation of energy consumption}

This experiment was performed to carry out an assessment of the energy demanded by the wireless card in the ad hoc node. In this case, the layout of experiment 2 was set up for the case of two nodes. In addition, the current sensor (INA219), located at the transmitter node, is activated. The sensor is installed as a current tester on the power cable of the wireless card and the captured data are transferred to the platform (Raspberry Pi) via an I2C bus. Table 6 summarizes the configuration parameters used in the evaluation.

Table 6. Wireless card, current sensor and traffic configuration to evaluate the energy consumption.

\begin{tabular}{l|l}
\hline Parameter & Description/Value \\
\hline Tx Power & $0 \mathrm{dBm}$ \\
Data Rate & $54 \mathrm{Mbps}$ fixed \\
Rx Sensitivity & $-76 \mathrm{dBm}$ \\
Anntena Gain & $5 \mathrm{dBi}$ \\
Traffic CBR (Iperf) & Duration: 10s 24Flows: 100k to 54 Mbps \\
Current Sensor (INA219) & Samples interval 1000us, 12 bit resolution \\
I2C Bus speed & $3 \mathrm{Mbps}$ \\
\hline
\end{tabular}

In Figure 22, examples of samples captured for different traffic flows are presented. It has been plotted an interval of 1.5 in order to visualize the behavior of the samples. Specifically, two levels of intensity captured by the sensor ( $409 \mathrm{~mA}$ and $204 \mathrm{~mA})$ can be seen. It can be seen that as traffic load increases, the number of samples with a value of $490 \mathrm{~mA}$ is higher. Consequently, this level of intensity can be attributed to the operation of the wireless card in transmission mode, while the level of $204 \mathrm{~mA}$ may be interpreted as baseline consumption generated jointly by the medium sense and the reception process.

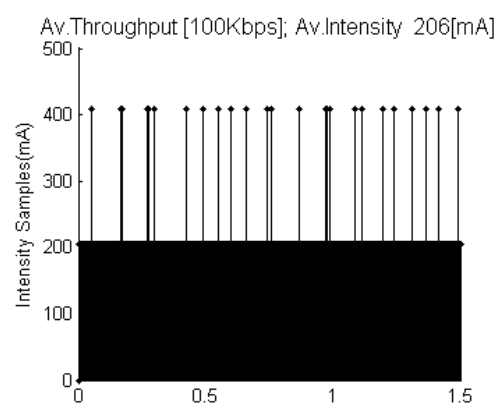

(a)

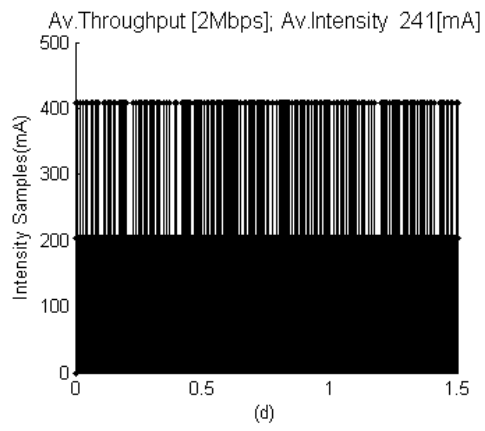

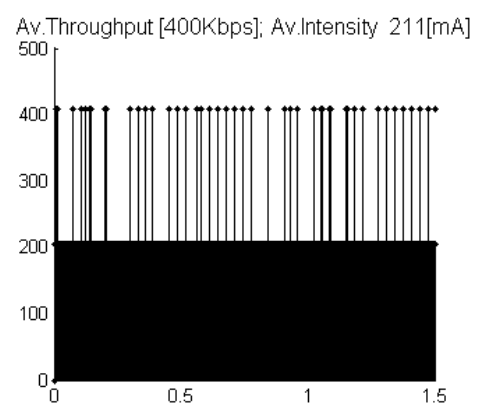

(b)

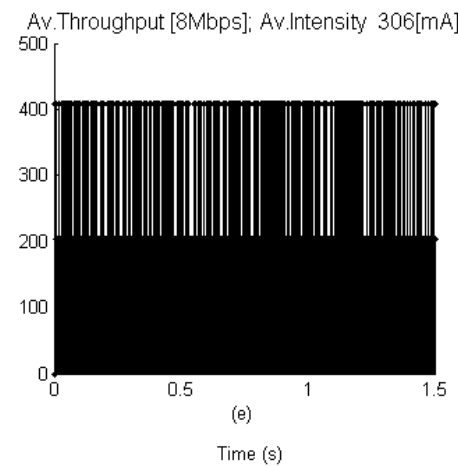

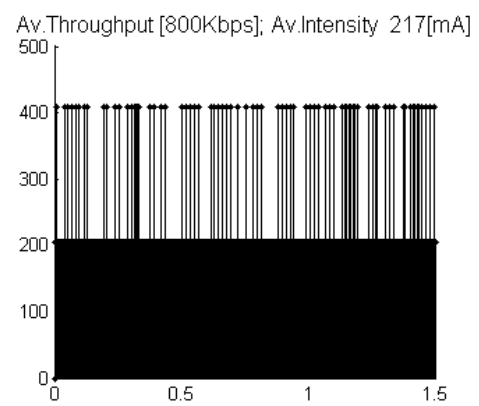

(c)

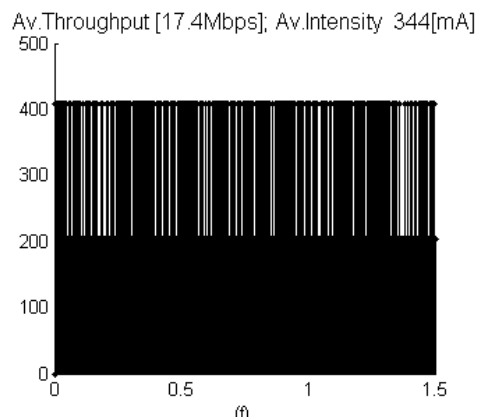

Figure 22. Intensity samples captured in the Tx Node for different traffic flows. (a) $100 \mathrm{Kbps}$, (b) $400 \mathrm{Kbps}$ (c) $800 \mathrm{Kbps}$,

(d) $2 \mathrm{Mbps}$, (e) $8 \mathrm{Mbps}$ and (f) 17.4Mbps. 
Such baseline consumption is dominant in experiments especially when the card operates at a reduced rate relative to maximum capacity; this is the case of the Figures 22 (a), (b) and (c) whose values of average intensity do not present major changes. On the other hand, Figures 22 (d), (e), (f) corresponding to the average throughput for 2,8 and $17 \mathrm{Mbps}$ show significant variation in the average intensity, particularly increases of $18 \%(241 \mathrm{~mA}), 50 \%$ $(306 \mathrm{~mA})$ and $68 \%(344 \mathrm{~mA})$, respectively.

In order to characterize such behavior, the percentage increment of intensity consumption is presented in Figure 23, starting at the base consumption level of the wireless card (204mA). As it can be seen, traffic load close to $20 \mathrm{Mbps}$ (maximum throughput) represents an increase of $70 \%(347 \mathrm{~mA})$ in the average intensity consumption. It is also worth noting the relationship between the consumption shown and the average throughput seen in Figure 18 , which both show the same linear trend until a steady maximum.

Additionally, Figure 23 highlights the relationship between video layers and intensity consumption. As it can be observed, non-adaptive transmission (layer 19) involves an increasing higher than $30 \%$ while the adapted flows present lower consumption levels ( $6 \%$ and $5 \%$ for layers 12 and 14, respectively). Layer 0 causes little variation in power consumption. Therefore, results show that an adaptive bitrate scheme provides an important reduction in energy consumption and the duration of the battery life of ad hoc nodes.

$\cdots$ - Increase $(\%)$

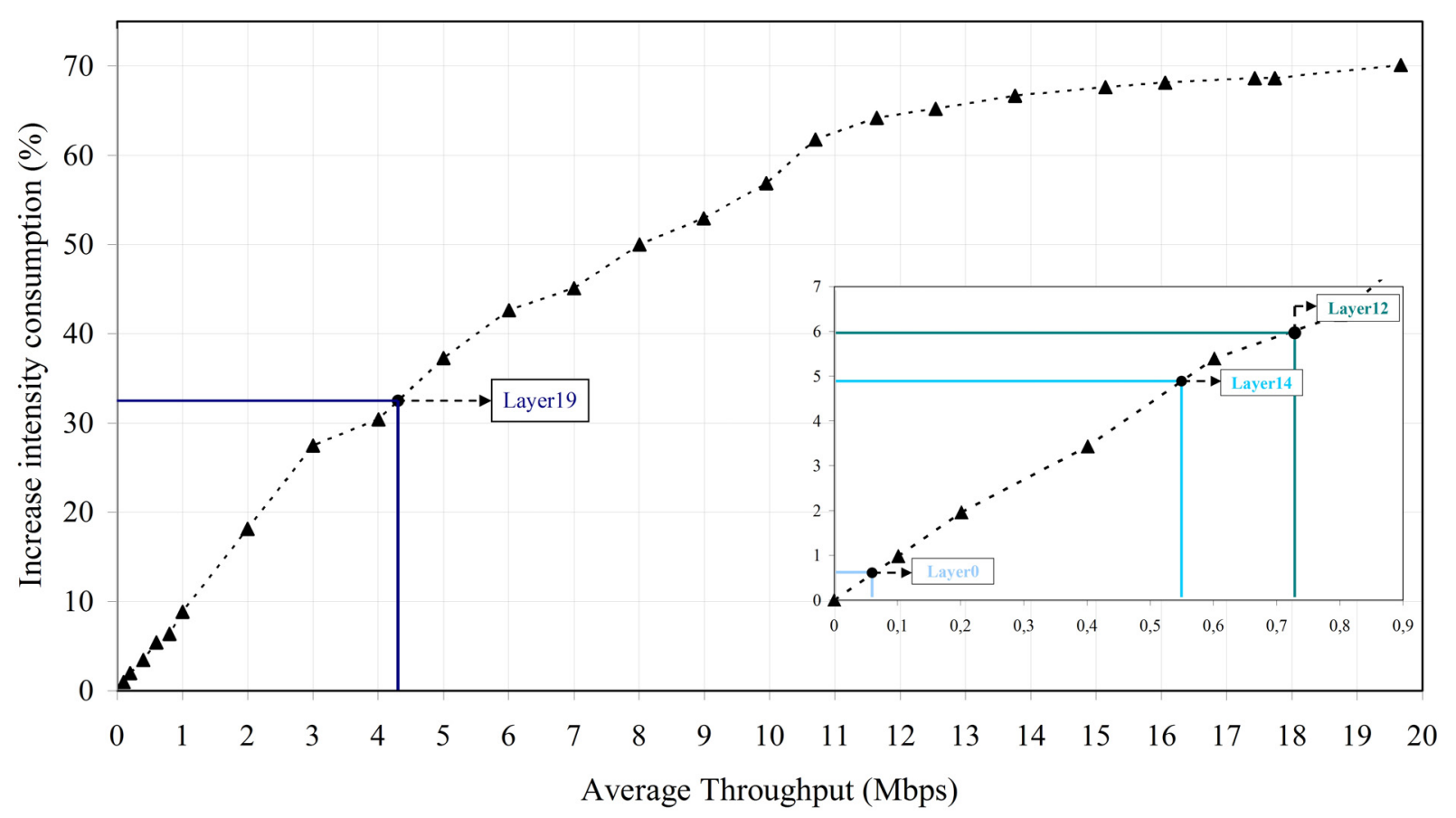

Figure 23 Comparison between the increase of the intensity consumption and the effective throughput transmission.

\section{CONCLUSIONS}

In this paper, two transmission schemes of SVC video streams over mobile ad hoc networks are analyzed. The proposed strategy for adaptive transmission of encoded video using SVC, in which the video source is able to adjust bit rate according to available bandwidth, is evaluated against the traditional strategy of transmission of video streams, in which the source sends data at a constant rate. In order to provide mobile ad hoc networks with the necessary mechanisms to handle a certain level of QoS, as well as an inter-layer mechanism to let applications determine the availability of network resources, a novel routing protocol for mobile networks previously developed was used, which is oriented to multimedia transmission. A simulation platform that allows developing 
experiments involving SVC video transmission over ad hoc mobile networks is also implemented. The integration of these solutions into the network simulator NS- 2 provides an efficient platform to explore new transmission schemes, as well as integration with other adaptive protocols, such as DCCP and TRFC.

The findings of the simulations show that adaptive strategy reduces network congestion and packet loss by reducing the transmission rate according to the change in available bandwidth. This adaptation is done by removing or adding layers of SVC video stream. After assessing the quality of received video, results show that although using the adaptive scheme less video data is transmitted, received quality is better than sending all video layers at a higher transmission rate. It has also been shown that the integration of this simulation platform and the formerly developed routing protocol form a useful tool for studying the SVC video transmission over ad hoc mobile networks. The developed simulation platform is publicly available and can be downloaded from the following link [45]. Additionally, we presented the design and implementation of a testbed in order to evaluate the proposal. In that sense a set of experiments were performed to characterize the multi-hop scenario, specially the throughput behavior in a linear topology with variable length. However, the greatest difficulties appeared in the emulation of the mechanism of adaptation. In particular, we detected that the average bitrate is not a suitable parameter in order to select the adapted data flows; it due to the bursts present in a video generate a load of traffic that exceed significantly the average value. Therefore, a detailed analysis of the video profile is required in order to prevent this behavior. In particular, the maximum bitrate can be a more suitable parameter. However, despite of this consideration, there are not guarantees against variations in the throughput in the real environment that allow maintain an accurate control in the experiments. Moreover, we performed and experiment in order to characterize the current consumption that demands the wireless card as a throughput function. Results were analyzed in relation with the bitrate adapted mechanism and show this strategy contributes to maintain a minimal increment in the intensity level during the wireless card operation.

Finally, as future work we plan to analyze the implementation of an adaptation mechanism that allows a suitable selection of the video flows such as the AQA-AODV scheme described in the proposal. Likewise, it is planned to complement the analysis of video transmission over ad hoc networks, performing a subjective quality assessment of received videos (QoE, Quality of Experience).

\section{ACKNOWLEDGEMENTS}

This paper was performed with the support of the National Secretary of Higher Education, Science, Technology and Innovation (SENESCYT)-Ecuador Government (scholarship 195-2012) and the Multimedia Communications Group (COMM) belong to the Institute of Telecommunications and Multimedia Applications (iTEAM)Universitat Politècnica de València.

\section{REFERENCES}

[1] M. Conti and S. Giordano, "Mobile ad hoc networking: milestones, challenges, and new research directions," IEEE Commun. Mag., vol. 52, no. 1, pp. 85-96, Jan. 2014.

[2] "AVC : Advanced video coding for generic audiovisual services," ITU-T 2013. Rec. H.264 \& ISO/IEC 14496-10. .

[3] A. Lady, "Introducing the Raspberry Pi 2 - Model B," 2015. [Online]. Available: https://learn.adafruit.com/downloads/pdf/introducing-theraspberry-pi-2-model-b.pdf.

[4] J. Famaey, S. Latre, N. Bouten, W. Van de Meerssche, B. De Vleeschauwer, W. Van Leekwijck, and F. De Turck, "On the merits of SVC-based HTTP Adaptive Streaming," Integrated Network Management (IM 2013), 2013 IFIP/IEEE International Symposium on. pp. 419-426, 2013.

[5] H. Kalva, V. Adzic, and B. Furht, "Comparing MPEG AVC and SVC for adaptive HTTP streaming," in 2012 IEEE International Conference on Consumer Electronics (ICCE), 2012, pp. 158-159.

[6] S. Xiang, L. Cai, and J. Pan, "Adaptive scalable video streaming in wireless networks," in Proceedings of the 3rd Multimedia Systems Conference on - MMSys '12, 2012, pp. 167-172.

[7] "Information technology -Dynamic adaptive streaming over HTTP (DASH) -- Part 1: Media presentation description and segment formats," Technical Report. ISO/IEC 23009-1:2014, 2014. .

[8] M. Sanna and E. Izquierdo, "Live scalable video streaming on peer-to-peer overlays with network coding," 2013 IEEE Latin-America Trans., vol. 11, no. 3, pp. 962-968, Nov. 2013.

[9] M. N. Alabdulkarim and N.-E. Rikli, "QoS Provisioning for H.264/SVC Streams over Ad-Hoc ZigBee Networks Using Cross-Layer Design," in 
2012 8th International Conference on Wireless Communications, Networking and Mobile Computing, 2012, pp. 1-8.

[10] E. Yaacoub, F. Filali, and A. Abu-Dayya, "QoE Enhancement of SVC Video Streaming over Vehicular Networks Using Cooperative LTE/802.11p Communications," IEEE J. Sel. Top. Signal Process., vol. 9, no. 1, pp. 37 - 49, Jun. 2014.

[11] P. McDonagh and C. Vallati, "Investigation of scalable video delivery using H. 264 SVC on an LTE network," in Wireless Personal Multimedia Communications (WPMC), 2011 14th International Symposium, 2011, pp. 1-5.

[12] M. Halloush, H. R. Al-Zoubi, Z. Al-Qudah, and O. Alkofahi, "The Performance of MANET Routing Protocols for Scalable Video Communication," Commun. Netw., vol. 05, no. 02, pp. 119-125, May 2013.

[13] O. Ben Rhaiem and L. C. Fourati, "Routing protocols performance analysis for scalable video coding (SVC) transmission over mobile ad-hoc networks," in IEEE International Conference on Signal and Image Processing Applications, 2013, pp. 197-202.

[14] C. Lal, V. Laxmi, and M. S. Gaur, "QoS-aware routing for transmission of H.264/SVC encoded video traffic over MANETs," in 2013 19th AsiaPacific Conference on Communications (APCC), 2013, pp. 104-109.

[15] D. Radu, J. Yi, and B. Parrein, "QoE enhancement for H.264/SVC video transmission in MANET using MP-OLSR protocol," ISIVC 2012, the 6th International Symposium on signal, Image, Video and Communications. pp. 1-4, 04-Jul-2012.

[16] W.-P. Lai and E.-C. Liou, "A novel packet priority scheme and mapping algorithm for scalable video over wireless ad-hoc networks," in 2013 22nd Wireless and Optical Communication Conference, 2013, pp. 366-371.

[17] T. A. Le and H. Nguyen, "End-to-end transmission of scalable video contents: performance evaluation over EvalSVC-a new open-source evaluation platform," Multimed. Tools Appl., vol. 72, no. 2, pp. 1239-1256, Apr. 2013.

[18] C.-H. Ke, “myEvalSVC-an Integrated Simulation Framework for Evaluation of H.264/SVC Transmission,” vol. 6, no. 1, pp. 377-392, 2012.

[19] B. Görkemli and A. M. Tekalp, “Adaptation strategies for MGS scalable video streaming," Signal Process. Image Commun., vol. 27, no. 6, pp. 595-611, Jul. 2012.

[20] H. Schwarz, D. Marpe, and T. Wiegand, "Overview of the scalable video coding extension of the H. 264/AVC standard," IEEE Trans. Circuits Syst. Video Technol., vol. 17, no. 9, pp. 1103-1120, 2007.

[21] V. Carlo, O. Victor, and M. Prasant, "Experimental work versus simulation in the study of mobile ad hoc networks," in Mobile Ad Hoc Networking: Cutting Edge Directions, Second Edition, 2013, pp. 191-238.

[22] E. Nordstrom, P. Gunningberg, and H. Lundgren, "A Testbed and Methodology for Experimental Evaluation of Wireless Mobile Ad hoc Networks," in First International Conference on Testbeds and Research Infrastructures for the DEvelopment of NeTworks and COMmunities, 2005, pp. 100-109.

[23] J. Yi, A. Adnane, S. David, and B. Parrein, "Multipath optimized link state routing for mobile ad hoc networks," Ad Hoc Networks, vol. 9, no. 1, pp. 28-47, Jan. 2011.

[24] J. Toutouh and E. Alba, "Light commodity devices for building vehicular ad hoc networks: An experimental study," Ad Hoc Networks, vol. 37, pp. 449-511, 2016.

[25] G. Anastasi, E. Borgia, and M. Conti, "Understanding the real behavior of Mote and 802.11 ad hoc networks: an experimental approach," Pervasive Mob. Comput., vol. 1, pp. 237-256, 2005.

[26] J. David, S. Tim, F. Russ, S. Daniel, Montrallo Flickinger Leigh, R. Robert, and L. Jay, "Mobile Emulab: A Robotic Wireless and Sensor Network Testbed," INFOCOM 2006, 2006. .

[27] D. Wu, D. Gupta, and P. Mohapatra, "QuRiNet: A wide-area wireless mesh testbed for research and experimental evaluations," Ad Hoc Networks, vol. 9, no. 7, pp. 1221-1237, 2011.

[28] H. Soroush, N. Banerjee, A. Balasubramanian, M. D. Corner, B. N. Levine, and B. Lynn, "DOME: a diverse outdoor mobile testbed," HotPlanet '09 Proc. 1st ACM Int. Work. Hot Top. Planet-Scale Mobil. Meas., pp. 1-6, 2009.

[29] A. Rice and S. Hay, "Measuring mobile phone energy consumption for 802.11 wireless networking," Pervasive Mob. Comput., vol. 6, no. 6, pp. 593-606, 2010.

[30] L. Feeney and M. Nilsson, "Investigating the Energy Consumption of a Wireless Network Interface in an Ad Hoc Networking Environment," pp. 1557, 1548, 2001.

[31] S. Keranidis, G. Kazdaridis, V. Passas, T. Korakis, I. Koutsopoulos, and L. Tassiulas, "Online energy consumption monitoring of wireless testbed infrastructure through the NITOS EMF framework," Proc. 8th ACM Int. Work. Wirel. Netw. testbeds, Exp. Eval. Charact. - WiNTECH '13, p. 73, 2013.

[32] T. Thang, H. Le, and H. Nguyen, “Adaptive video streaming over HTTP with dynamic resource estimation,” J. Commun. Networks, vol. 15, no. 6, pp. 635-644, 2013.

[33] K. Evensen, T. Kupka, H. Riiser, and P. Ni, "Adaptive media streaming to mobile devices: challenges, enhancements, and recommendations," Adv. Multimed., 2014.

[34] T. Christian, G. Carsten, B. Ali, S. Thomas, and G. Bernd, “Adaptive Media Streaming," IEEE Journal of Selected Areas in Communications, 2014. .

[35] Z. Ce and L. Yuenan, Advanced Video Communications over Wireless Networks. Boca Raton: CRC Press, 2013.

[36] K. Birkos, C. Tselios, T. Dagiuklas, and S. Kotsopoulos, "Peer selection and scheduling of H.264 SVC video over wireless networks," in 2013 IEEE Wireless Communications and Networking Conference (WCNC), 2013, pp. 1633-1638.

[37] W. Castellanos, P. Acelas, P. Arce, and J. C. Guerri, "Evaluation of a QoS-Aware Protocol with Adaptive Feedback Scheme for Mobile Ad Hoc Networks," in Quality of Service in Heterogeneous Networks, 2009, pp. 120-127.

[38] Moving Pictures Experts Group and ITU-T Video Coding Experts Group, “H. 264/SVC reference software (JSVM 9.19.14) and Manual.” Moving Pictures Experts Group and ITU-T Video Coding Experts Group, 2011. 
[39] Xiph.org video test media, "CREW video sequence.” [Online]. Available: https://media.xiph.org/video/derf/y4m/crew_cif.y4m.

[40] ALFA Networks, "Datasheet- AWUS036NH 802.11n/b/g 2W Long-Rang USB Adapter," ALFA Networks, 2000. [Online]. Available: http://www.alfanetwork.co.nz/downloads/1410553/AWUS036NH.pdf.

[41] A. Lady, "Adafruit INA219 Current Sensor Breakout," 2015. [Online]. Available: https://learn.adafruit.com/downloads/pdf/adafruit-ina219current-sensor-breakout.pdf.

[42] Adafruit, "GitHub Adafruit INA219 Current Sensor." [Online]. Available: https://github.com/adafruit/Adafruit_INA219.

[43] Yuv Sequences Trace, "Big Bug Bunny video sequence.” [Online]. Available: http://trace.eas.asu.edu/yuv/bbb/BBB_CIF_24fps.gz.

[44] J. John, F. Sherelle, S. Roland, and S. Tim, “Medianet Reference Guide," $2010 . \quad$ [Online]. Available: http://www.cisco.com/c/en/us/td/docs/solutions/Enterprise/Video/Medianet_Ref_Gd/medianet_ref_gd.pdf.

[45] W. Castellanos, "SVCEval-RA - An Evaluation Framework for Adaptive Scalable Video Streaming," SourceForge Project, 2014. [Online]. Available: http://sourceforge.net/projects/svceval-ra/.
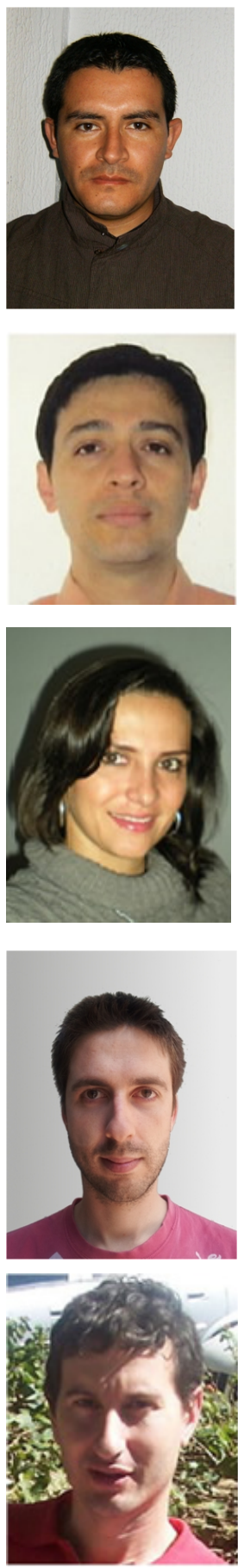

Santiago González Martínez, received the Electronic Engineering degree from the Universidad Politécnica Salesiana (UPS), Cuenca- Ecuador, in 2006 and M.S Degree in Tecnologías Informáticas Avanzadas, from Universidad Castilla-La Mancha, Ciudad Real, Spain, 2009. From 2010 to 2013 he worked as professor at Electronic and Telecommunications Engineering SchoolUniversidad de Cuenca (Ecuador). Currently, he is a Researcher at the Multimedia Communications research group (COMM) of the Institute of Telecommunications and Multimedia Applications (iTEAM), UPV, where he is working toward the Ph.D. degree. His research interests include Energy Efficiency and QoS on Wireless Ad-Hoc Networks.

Wilder Castellanos received his Electronic Engineering degree from Industrial University of Santander, Bucaramanga, Colombia, in 2002 and the M.Sc. Telematics and the Ph.D degree in Telecommunications from the Universitat Politècnica de València (UPV), Spain in 2009 and 2015 respectively. His current research interests include QoS-aware architectures and multimedia streaming in mesh/ad hoc networks.

Paola Guzmán Castillo received her Electronic Engineering degree and the M.Sc. degree in Electronic Engineering from Industrial University of Santander, Bucaramanga, Colombia, in 2001 and 2005 respectively. From 2006 to 2010 she worked as professor at St. Thomas University and Industrial University of Santander, Bucaramanga, Colombia. In 2014 she received her M.S. in Telematics from the Universitat Politècnica de València (UPV), Spain. Currently, she is a researcher at the Multimedia Communications research group (COMM) of the Institute of Telecommunications and Multimedia Applications (iTEAM), UPV, where she is working towards the Ph.D. degree. Her current research interests include QoS, QoE and adaptive transport of 3D video over IP networks.

Pau Arce, received his Telecommunications Engineering degree, M.S. in Telematics and the Ph.D degree in Telecommunications from the Universitat Politècnica de València (UPV), Spain, in 2005, 2007 and 2014 respectively. Currently he works as a researcher at the Institute of Telecommunications and Multimedia Applications (iTEAM). His research interests include multimedia QoS, routing on wireless ad hoc networks and performance evaluation of computer systems.

Juan Carlos Guerri, received the M.S. and Ph.D. (Dr.Ing.) degrees, both in telecommunication engineering, from the Universitat Politècnica de València (UPV), Valencia, Spain, in 1993 and 1997, respectively. He is a Professor in E.T.S. Telecommunications Engineering at the UPV, where he leads the Multimedia Communications research group (COMM) of the iTEAM Institute. He is currently involved in research and development projects for the application of multimedia to industry, medicine, education, and communications. 